\title{
The low-energy concentrator spectrometer on-board the BeppoSAX X-ray astronomy satellite
}

\author{
A.N. Parmar, D.D.E. Martin, M. Bavdaz, F. Favata, E. Kuulkers, G. Vacanti, U. Lammers, A. Peacock, \\ and B.G. Taylor \\ Astrophysics Division, Space Science Department of ESA, ESTEC, Postbus 299, 2200 AG Noordwijk, The Netherlands
}

Received May 15; accepted July 2, 1996

\begin{abstract}
The payload of the Italian/Dutch spacecraft BeppoSAX includes a set of four X-ray concentrators each of geometric area $124 \mathrm{~cm}^{2}$ together with imaging gas scintillation proportional counter detectors located at the focal planes. One of these detectors, the Low-Energy Concentrator Spectrometer (LECS), is sensitive to X-rays in the energy range $0.1-10 \mathrm{keV}$, while the other three cover a narrower energy range of $1.3-10 \mathrm{keV}$. In order to achieve the extended low-energy response of the LECS a novel type of gas scintillation proportional counter has been developed which dispenses with the separate drift and scintillation regions of conventional instruments. The design and performance of the instrument together with its calibration and data analysis system are described here.
\end{abstract}

Key words: instrumentation: detectors - methods: data analysis - methods: observational — techniques: spectroscopic — X-rays: general

\section{Introduction}

BeppoSAX is an Italian/Dutch spacecraft that was placed into low-Earth orbit by an Atlas I launch vehicle on 1996 April 30. The nominal orbit is circular at a height of $600 \mathrm{~km}$ with an inclination of $<4^{\circ}$ to the equator in order to take maximum advantage of the shielding of the Earth's magnetic field. During the expected lifetime of up to 4 years, BeppoSAX will make $2000-3000$ observations of cosmic X-ray sources over the unprecendently wide energy range of $0.1-300 \mathrm{keV}$. Descriptions of the mission are to be found in Spada (1983), Butler \& Scarsi (1990), Scarsi (1993) and Boella et al. (1996b). The scientific objectives of the mission include broad band spectroscopy in the energy range $0.1-10 \mathrm{keV}$ with imaging resolution of $1^{\prime}$, nonimaging spectroscopy in the energy range $3-300 \mathrm{keV}$ and

Send offprint requests to: A.N. Parmar:

aparmar@astro.estec.esa.nl variability studies of X-ray source intensities and spectra on timescales from milliseconds to months (Perola 1983). Mission operations will be conducted from Rome, Italy and are designed to allow a flexible response to any targets of opportunity.

The payload consists of the Phoswich Detector System (PDS), the High Pressure Gas Scintillation Proportional counter (HPGSPC), two Wide Field Cameras (WFC), three imaging Medium Energy Concentrator Systems (MECS) and the imaging Low Energy Concentrator System (LECS). All the instruments are coaligned except for the WFCs which point in opposite directions along an axis perpendicular to the other instruments. Each imaging GSPC is located at the focal plane of an X-ray concentrator system. In order to extend the sensitive range of the imaging spectrometers to $<1.3 \mathrm{keV}$, the LECS dispenses with the separate drift and scintillation regions of conventional GSPCs and utilizes an extremely thin entrance window. This instrument was provided by SSD and is described here. Its primary objective is to provide better energy resolution at low energies than currently available non-dispersive detectors and so to open up new areas of astrophysical interest.

The last few years have been an exciting time for X-ray astronomy with the launches of the ROSAT, ASCA and XTE satellites. ROSAT provides high sensitivity and spatial resolution in the $0.1-2.5 \mathrm{keV}$ energy range, but with only moderate spectral resolution (Trümper 1983). XTE concentrates on timing studies in the $2-200 \mathrm{keV}$ energy range with moderate spectral resolution (Swank et al. 1995). The ASCA payload consists of both imaging CCD and GSPC detectors (Tanaka et al. 1994). The CCD detectors provide an energy resolution, $\Delta E$, of $100-150 \mathrm{eV}$ over the $\sim 0.5-10 \mathrm{keV}$ energy range, while the GSPCs provide $\Delta E \sim 500 \mathrm{eV}$ at $6 \mathrm{keV}$ over the energy range $1.0-10 \mathrm{keV}$. The good spectral and spatial resolutions, robustness and low power requirements of CCD detectors have ensured that they have become the preferred detectors for future X-ray spectroscopic investigations (Weisskopf 1987; Bignami et al. 1990; Wells et al. 1992). While their 
development is still proceeding, it is worth comparing their properties with those of GSPCs:

- The resolution of a typical GSPC detector at $6 \mathrm{keV}$ of $500 \mathrm{eV}$ is a factor $\sim 4$ worse than a CCD. However, the resolution, scales approximately as $E^{1 / 2}$ and so becomes comparable to that of CCDs at low $(\$ 0.5 \mathrm{keV})$ energies.

- GSPC detectors provide extremely good time resolution of up to a few $\mu$ s. This should be compared with a time of $\sim 2 \mathrm{~s}$ needed to read out a typical CCD. CCD time resolution can be improved to tens of $\mathrm{ms}$ by continuously reading the device. The spatial distribution of the observed X-rays then represents timing information.

- In X-ray astronomy CCD detectors are used in photon counting mode. This means that the mean arrival rate should be $<0.1$ photons pixel ${ }^{-1}$ frame $^{-1}$ which can place severe constraints on the maximum source strength allowed. The maximum count rate of the LECS is limited by the electronics to be $<2000$ counts s ${ }^{-1}$.

- The energy resolution of GSPCs is known to be extremely stable, whereas there are still concerns as to the effects of large radiation doses on CCDs (e.g., Holland et al. 1990).

- GSPCs have good background rejection capabilities using a variety of techniques over and above those used by CCDs.

\section{LECS description}

The LECS consists of Mirror, Detector, Electronics and Gas Units. The Mirror Unit is provided by the Italian Space Agency (ASI). The major components of the SSD provided units are shown in Fig. 1 and the overall performance is summarized in Table 1.

\subsection{Mirror unit}

A total of four flight Mirror Units (MUs) and one qualification model have been produced. The LECS uses unit FM3. Each MU consists of 30 nested, Au coated, confocal mirrors with a double cone approximation to Wolter I geometry (Citterio et al. 1985). The geometric area is $124 \mathrm{~cm}^{2}$ and the mirror diameters range from 16.2 to $6.8 \mathrm{~cm}$. The shell thicknesses range from 0.4 to $0.2 \mathrm{~mm}$ and the nominal focal length is $185 \mathrm{~cm}$. The total length of each mirror shell is $30 \mathrm{~cm}$. The mirrors were produced using a replication technique by nickel electroforming from super-polished mandrels. The calibration and performance of the flight MUs is described in Conti et al. (1994).

In order to deflect any plasma that might pass through the MU, a plasma protection grid is mounted on the underside of the unit. This consists of a fine pitched Aucoated $\mathrm{W}$ grid. In flight, the grid will be placed at $+28 \mathrm{~V}$
Table 1. LECS overall performance summary

\begin{tabular}{ll}
\hline Parameter & Value \\
\hline Energy Range & $0.1-10.0 \mathrm{keV}$ \\
Energy Resolution at $0.28 \mathrm{keV}$ & $32 \% \mathrm{FWHM}$ \\
Energy Resolution at $6 \mathrm{keV}$ & $8.8 \%$ FWHM \\
Angular Resolution at $0.28 \mathrm{keV}$ & $9.7 \mathrm{FWHM}$ \\
Angular Resolution at $6 \mathrm{keV}$ & $2.1 \mathrm{FWHM}$ \\
Effective Area at $0.28 \mathrm{keV}$ & $22 \mathrm{~cm}^{2}$ \\
Effective Area at $6 \mathrm{keV}$ & $50 \mathrm{~cm}^{2}$ \\
Field of view & $37^{\prime} \mathrm{diameter} \mathrm{circular}^{\prime}$ \\
Entrance window size & $20 \mathrm{~mm}$ diameter $^{-1}$ \\
Image scale & $1.86 \mathrm{~mm}^{-1}$ \\
Time Resolution & $16 \mu \mathrm{s}$ \\
Maximum Throughput & 2000 events s \\
Energy Spectrum & 1024 channels \\
Image size & 256 by 256 pixels \\
Pixel size & $14^{\prime \prime} 0$ by $14^{\prime \prime} .0$
\end{tabular}

potential, in order to shield the detector from ion impingement.

\subsection{Detector unit}

The Detector Unit (DU) consists of a gas cell, a photomultiplier tube (PMT), Front End Electronics (FEE) and two high-voltage supplies. The detector is protected by an $\mathrm{Al}$ cover on which a shutter mechanism is mounted. The shutter will be opened once in orbit. The detector is protected against space plasma ingress by a protection window located just beneath the shutter and by the MU plasma protection grid described in Sect. 2.1. The protection window consists of two layers of Polyimide with a total thickness of $200 \mathrm{~nm}$, separated by a layer of AlN and coated with a layer of AlN and $\mathrm{C}$ on the external surfaces (Fig. 2). Mechanical strength is provided by a $250 \mu \mathrm{m}$ hexagonal Polyimide grid. The top cover has a thin Be disk mounted on its interior upper surface in order to minimize any X-ray background induced by electron emission from the gas cell top disk which is at a potential of $-20 \mathrm{kV}$.

The detector also contains two ${ }^{55} \mathrm{Fe}$ radioactive sources which constantly illuminate regions of the detector outside the field of view (FOV), allowing the position and energy gains of the instrument to be continuously monitored. Each source produces Mn $K_{\alpha}$ and $K_{\beta}$ X-rays (5.89 and $6.49 \mathrm{keV}$, respectively) at a rate of 20 counts s${ }^{-1}$ in 1994 October, with a half-life of 2.73 years.

The method of operation of the detector is similar to that of a conventional GSPC (see Inoue et al. 1978; Davelaar et al. 1980; Peacock et al. 1980; Simons et al. 1985). An X-ray which is absorbed in the cell gas liberates a cloud of electrons. A uniform electric field between the entrance window kept at $-20 \mathrm{kV}$ and a grounded grid, 


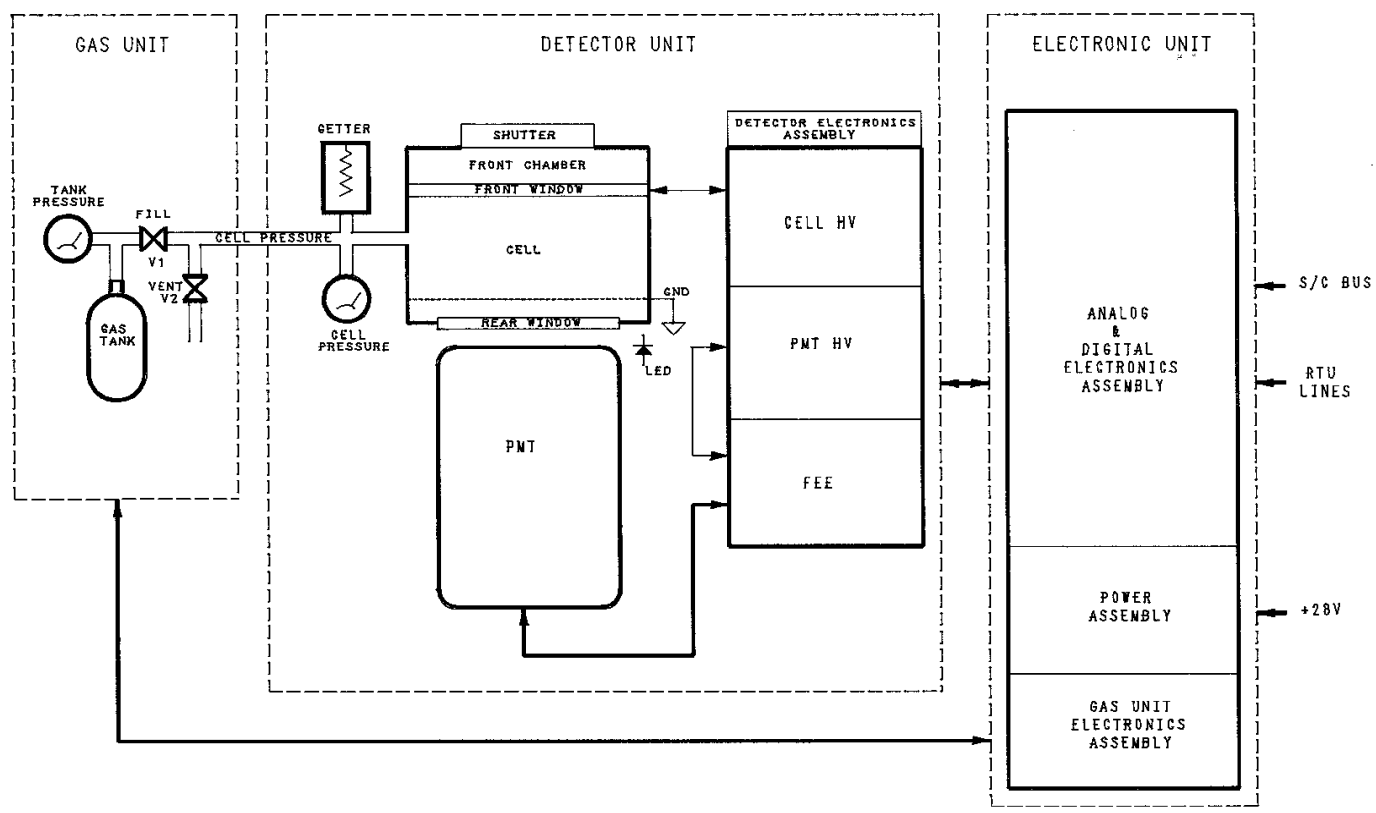

Fig. 1. LECS instrument schematic

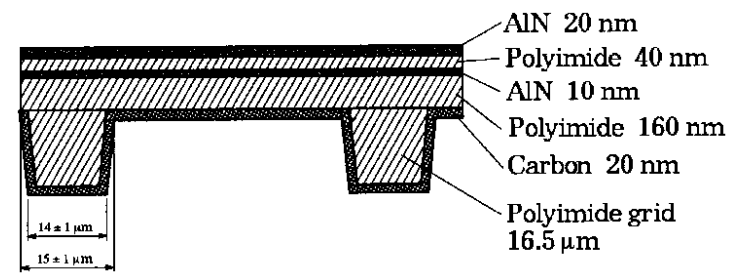

Fig. 2. Plasma protection window cross-section

causes scintillation of the Xe gas as the electrons travel towards the grid. The UV light from these scintillations is detected by the PMT mounted at the rear of the cell. The amount of light produced is proportional to the energy of the incident X-ray but, unlike a conventional GSPC, also depends on the depth at which the X-ray photon was absorbed. This depth is determined by measuring the duration of the scintillation light and is referred to as the "Burstlength". Thus an X-ray absorbed deep within the gas cell will have a shorter burstlength than one absorbed directly below the entrance window. The negative potential on the window and the high electric field ensure that electrons created by X-rays absorbed just beneath the entrance window have a high probability of entering the scintillation process.

The gas cell is a $11.4 \mathrm{~cm}$ diameter ceramic cylinder of depth $5 \mathrm{~cm}$ with a metallic top disk and lower flange. The PMT is mounted $1 \mathrm{~mm}$ from the quartz gas cell exit window. The cell is filled with Xe at a nominal pressure of 1.1 bar at $20{ }^{\circ} \mathrm{C}$. GSPCs are extremely sensitive to impurities and a getter is included to passively absorb small amounts of gas impurities. The getter can be activated by a current source once it has reached saturation. The gas cell has an extremely thin multi-layer entrance window consisting of three layers of Polyimide separated by $\mathrm{Al} / \mathrm{AlN}$ multilayers. This construction was chosen to minimize the leak rate and sensitivity to atomic oxygen corrosion. The nominal thickness of the window is given in Table 4 and its cross-section is shown in Fig. 3. The Al and AlN layers act as gas permeation barriers while the Polyimide provides mechanical support. The strength to support a differential pressure of over 1 bar is provided by a fine grid and a strongback. The strongback is made of $50 \mu \mathrm{m}$ thick $\mathrm{W}$ bars with a height of $3 \mathrm{~mm}$ and a pitch of $2.2 \mathrm{~mm}$ (Fig. 4). The fine grid consists of $25 \mu \mathrm{m}$ thick W foil which subdivides each strongback square into a matrix of 8 by 8 squares (Fig. 5 ).

The high voltage power supplies provide power to the gas cell and to the PMT. The cell voltage is commandable in 31 steps between -12 and $-20 \mathrm{kV}$ and that of the PMT between 0.8 and $1.5 \mathrm{kV}$ in 255 steps. The power supplies are based on a fly-back converter followed by an 8 stage Cockcroft-Walton multiplier. The output voltages are then filtered by a triple resistive-capacitive network to produce less than $0.1 \%$ (peak-to-peak) ripple.

The PMT is a ruggedized Hamamatsu R5218 tube with a $2.8 \mathrm{~mm}$ thick quartz entrance window of $76 \mathrm{~mm}$ diameter contained in a mu-metal housing to shield it from external magnetic fields. Nine anodes, each $13 \times 13 \mathrm{~mm}^{2}$ are positioned in a $3 \times 3$ square configuration with a separation of $1 \mathrm{~mm}$ as shown in Fig. 6. The anodes are separately read out and operated as an Anger camera (Anger 1958) to provide spatial information. The cathode is a special bialkali with a spectral response from 160 to $600 \mathrm{~nm}$. Amplification is provided by 15 proximity mesh dynodes. 


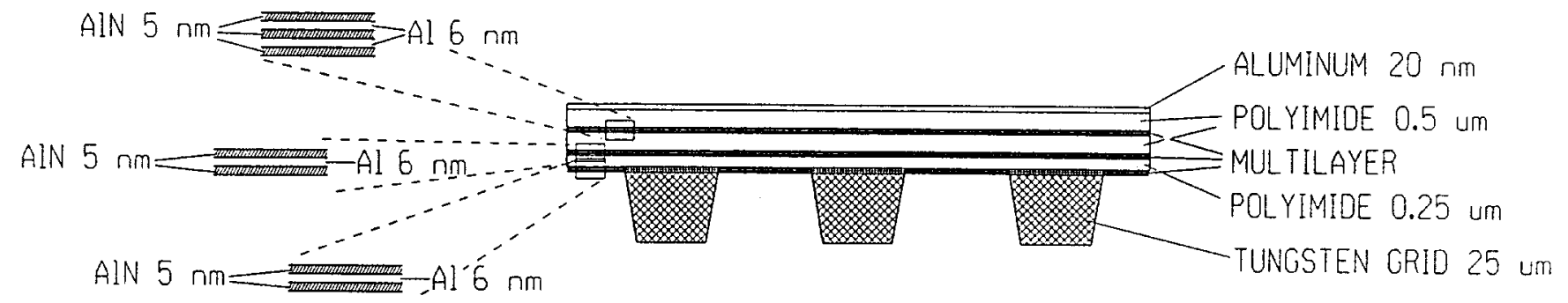

Fig. 3. Detector entrance window cross-section

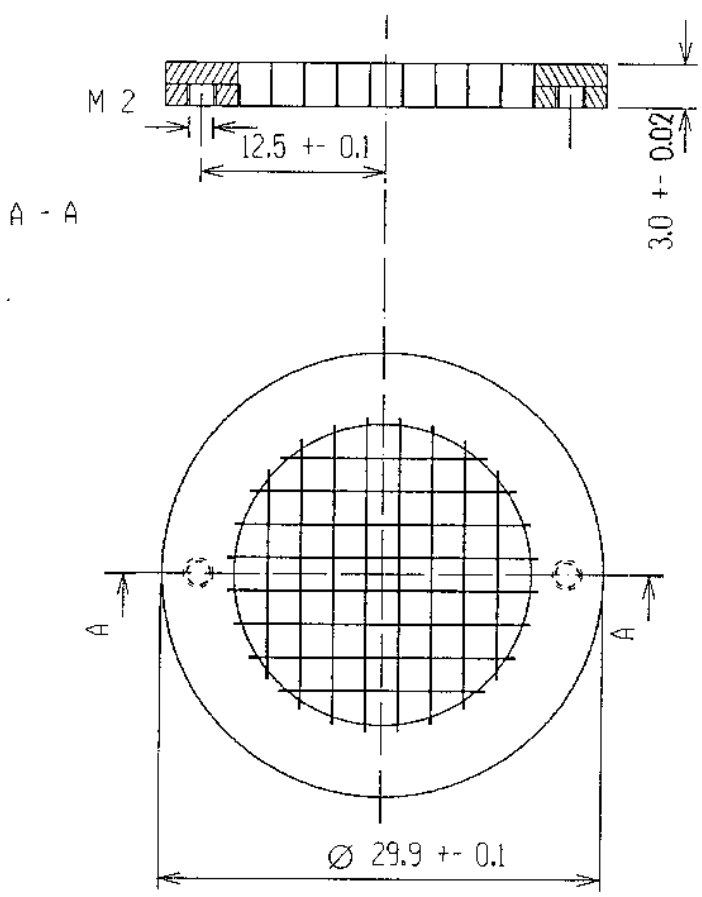

Fig. 4. Entrance window strongback structure

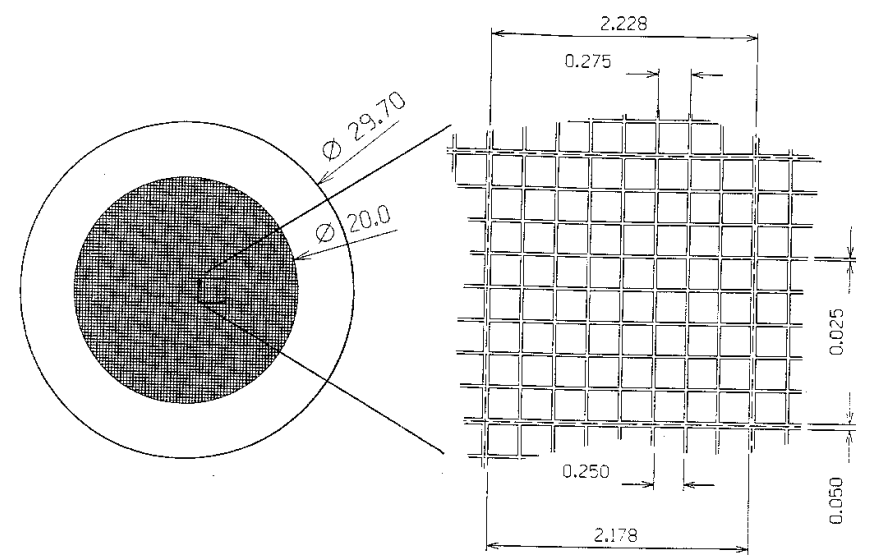

Fig. 5. Entrance window support grid structure

\begin{tabular}{|c|c|c|}
\hline 3 & 2 & 1 \\
\hline 6 & 5 & 4 \\
\hline 9 & 8 & 7 \\
\hline
\end{tabular}

Fig. 6. PMT anode layout. The above view is looking in the direction of the concentrators through the PMT. The arrows indicate the spacecraft physical axes

Within the FEE, each of the signals from the nine PMT anodes are fed to separate charge-sensitive amplifiers with a $60 \mu$ s time constant. The amplified signals are then combined to provide the energy $\left(S_{\mathrm{a}}\right), X\left(S_{x}\right)$ and $Y$ $\left(S_{y}\right)$ positions and veto $\left(S_{\mathrm{v}}\right)$ signals. Defining $A_{i}$ to be the output of the $i^{\text {th }}$ anode's pre-amplifier, gives:

$$
\begin{aligned}
S_{\mathrm{a}} & =\sum_{i=1}^{9} A_{i} \\
S_{x} & =A_{1}+A_{4}+A_{7}-A_{3}-A_{6}-A_{9}+0.5 S_{\mathrm{a}} \\
S_{y} & =A_{1}+A_{2}+A_{3}-A_{7}-A_{8}-A_{9}+0.5 S_{\mathrm{a}} \\
S_{\mathrm{v}} & =A_{5} .
\end{aligned}
$$

When divided by the $S_{\mathrm{a}}$ signal, the veto signal, $S_{\mathrm{v}}$, provides a measure of the light distribution in the detector. This signal has a high value for events that occur close to the center of the FOV and a low one for events that occur outside the FOV. The veto signal can also be used to distinguish between particle events (which typically appear extended and therefore have low veto values) and on-axis $\mathrm{X}$-ray events. The four signals are buffered and passed to the Electronics Unit (EU) for further processing.

The DU also includes a pressure transducer for monitoring the cell pressure, a thermistor to monitor the PMT temperature, two micro-switches to determine the open and closed position of the shutter mechanism and analog monitors of the two high voltage outputs.

\subsection{Electronics unit}

The EU provides the electrical interface between the instrument and the spacecraft. The main micro-processor is 
a radiation-tolerant $80 \mathrm{C} 86$ with $64 \mathrm{k}$-Bytes of data $\mathrm{RAM}$ and $32 \mathrm{k}$-Bytes of program memory. An additional $32 \mathrm{k}$ Bytes of RAM is available for software updates. A second processor is dedicated to communications with the spacecraft using the ESA On-Board Data Handling protocol. Communication between the two processors is via a dual port RAM.

The EU performs many functions. The analog signals provided by the FEE pass through optimized pulse shapers with a $5.6 \mu$ s time constant and pole-zero cancelation of the FEE's $60 \mu$ s time constant. Additionally, the energy signal is fed through a $1.5 \mu$ s pulse shaper for burstlength determination. The output of the filters are sampled at their peaks and the position, veto and burstlength signals normalized using the energy signal. At this stage, qualification of the signals occurs, based on programmable amplitude windows. In the case of a qualified event, the signals are converted to digital values using Wilkinson converters which provide good integral and differential linearity. The measured differential nonlinearity of the energy channel is $<3.5 \%$ of the least significant bit. The energy channel, $S_{\mathrm{a}}$, is converted to a 10 bit value and is referred to as the PHA signal. The other channels are converted to 8 bit values and are referred to as the RAWX, RAWY, VETO, and BL signals (coming from the $S_{x}, S_{y}, S_{\mathrm{v}}$ signals and the burstlength determination circuitry, respectively). Between the time the EU detects an event and its conversion, the acquisition chain is inhibited to prevent subsequent pulses from affecting the current measurement. The time the instrument is inhibited in this way is accumulated in a deadtime counter. Whenever a non-qualified event is detected, the analog-todigital conversion process is aborted in order to minimize the deadtime. The deadtime for each event is amplitude dependent, and is in the range $64 \mu \mathrm{s}$, for non-qualified events, to $250 \mu$ s for full-scale events.

The on-board software is written in "C" with assembler language used for time critical tasks. The system runs under the control of a real-time operating system, which schedules the tasks for instrument monitoring and protection, command processing and execution, data processing and packetization. Event processing and telemetry transmission are interrupt triggered. Whenever a qualified event is detected, the analog electronics processes the different signals and converts the analog signals into digital values as described above. An on-board counter, synchronized to the spacecraft's ultra-stable clock, time-tags the event with $16 \mu$ s resolution. The complete data set is then retrieved by the main processor and packetized for transmission to ground. Alternatively, the event data can be accumulated by the EU software into images and spectra in order to conserve telemetry usage.

\subsection{Gas unit}

The original specification on the leak rate of gas cell entrance window was sufficiently high to require a gas filling system. However, the leak rate of the final design is so low $\left(<10^{-6}\right.$ mbar liter $\left.\mathrm{s}^{-1}\right)$ that such a system is no longer required for this purpose. The GU was originally designed to autonomously maintain the gas cell pressure in a programmable range between 800 and 1200 mbar. However, this operation is no longer required and it is disabled under normal operations. Instead, the GU can be used to completely vent and refill the gas cell should the gas become contaminated. The unit consists of two identical 0.5 liter Ti reservoirs containing 20 bar liters of Xe, input and vent valves, a pressure transducer, a thermistor and the necessary structure and piping. The non-latching input valve can be actuated in pulse mode from the EU to allow gas to enter the cell. The pulse duration is programmable to minimize the number of actuations. The non-latching vent valve allows gas to escape from the cell into free space. The pressure transducer monitors the gas pressure in the reservoirs.

\section{Instrument performance}

Great care has been taken to ensure a good calibration of the LECS. Each of the critical components has been separately calibrated and then the entire calibration has been verified at instrument level. The measurements have been performed using SSD's 6 meter X-ray beam, the Berlin synchrotron facility (BESSY), and the PANTER long-beam X-ray facility of the Max Planck Institut für Extraterrestrische Physik near Munich. All measurements reported here were performed in vacuum with the nominal voltages of $-20 \mathrm{kV}$ on the cell and $1082 \mathrm{~V}$ on the PMT.

Initial functional tests were carried out using SSD's X-ray beam facility. During these tests, the LECS was mounted behind a pin-hole plate and illuminated with $\mathrm{Al}(1.49 \mathrm{keV})$ and $\mathrm{Fe}(6.41 \mathrm{keV})$ radiation from a source located 5 meters away. The pin-hole plate consists of a $130 \mu \mathrm{m}$ thick Ni sheet with $1 \mathrm{~mm}$ diameter holes in a rectangular grid with a $4 \mathrm{~mm}$ pitch. Figure 7 shows a representative image obtained with $\mathrm{Fe} \mathrm{K}_{\alpha}$ illumination to illustrate the overall performance of the LECS. The two ${ }^{55} \mathrm{Fe}$ calibration sources are visible in the upper left and lower right corners. The off-axis pin-hole images are asymmetric and extended towards the outside of the FOV. This is caused by the penetration of $\gtrsim 1.5 \mathrm{keV}$ X-rays into the cell gas before absorption. Because of the driftless design (see Sect. 2.2), these penetrating X-rays will give rise to lower PHA and BL values than those absorbed directly beneath the entrance window. In addition, the variation in viewing angle and footprint of the scintillation light will cause the measured position to be shifted. The dependence of mean penetration depth on energy under the LECS nominal operating conditions is shown in Fig. 8. 
Table 2. PANTER calibration lines

\begin{tabular}{llll}
\hline Line & $\begin{array}{l}\text { Energy } \\
(\mathrm{keV})\end{array}$ & Line & $\begin{array}{l}\text { Energy } \\
(\mathrm{keV})\end{array}$ \\
\hline $\mathrm{B} K_{\alpha}$ & 0.18 & $\mathrm{Ag} L_{\beta}$ & 3.26 \\
$\mathrm{C} K_{\alpha}$ & 0.28 & $\mathrm{Ti} K_{\alpha}$ & 4.52 \\
$\mathrm{O} K_{\alpha}$ & 0.53 & $\mathrm{Ti} K_{\beta}$ & 4.94 \\
$\mathrm{Cu} L_{\alpha}$ & 0.93 & $\mathrm{Cr} K_{\alpha}$ & 5.42 \\
$\mathrm{Al} K_{\alpha}$ & 1.49 & $\mathrm{Fe} K_{\alpha}$ & 6.41 \\
$\mathrm{Si} K_{\alpha}$ & 1.74 & $\mathrm{Cu} K_{\alpha}$ & 8.06 \\
$\mathrm{Ag} L_{\alpha}$ & 3.00 & & \\
\hline
\end{tabular}

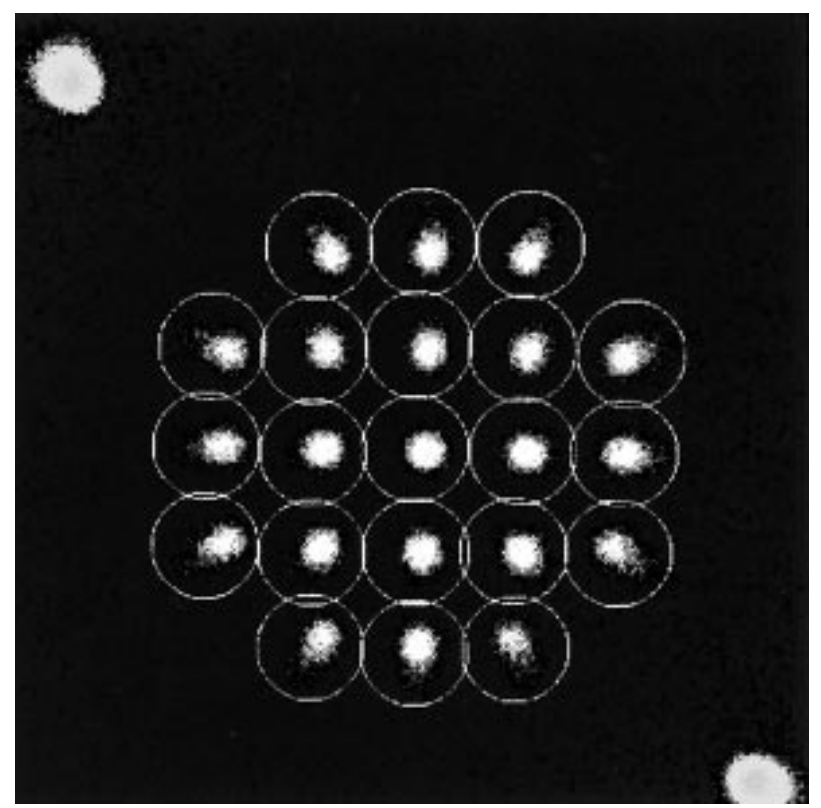

Fig. 7. Multi pin-hole array $6.41 \mathrm{keV}$ image. The calibration sources are the bright spots in the upper left and lower right corners. The circles indicate the regions used for gain mapping. The circular FOV of the LECS is approximately bounded by the outermost pin-holes

The effect of penetration on the LECS event data is further illustrated in Fig. 9. This shows BL versus energy for incident X-ray energies just above and just below the $\mathrm{Xe} L_{\mathrm{III}}$ edge, where there is a large discontinuity in mean X-ray absorption depth (see Fig. 8). At an energy of $4.77 \mathrm{keV}$ (i.e. just below the Xe $L_{\mathrm{III}}$ edge) the mean penetration depth is large and the extended tail of events in the upper panel of Fig. 9 towards lower energy and $\mathrm{BL}$ values (1) is caused by penetration. The events labeled as (2) are due to multiple events occurring within the EU sampling window i.e. event pile-up. This feature is prominent due to the high count rates used for these

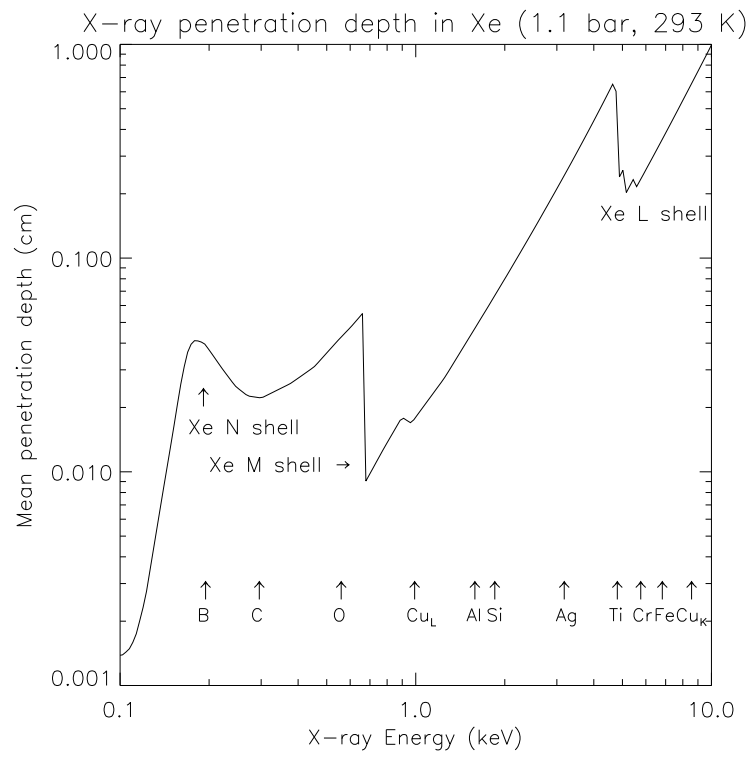

Fig. 8. Mean absorption depth in Xe as a function of X-ray energy using the absorption coefficients of Henke et al. (1993). The positions of the Xe absorption edges and the energies of the lines used in the LECS PANTER calibration are indicated

calibration measurements, but will be negligible for most astronomical sources. In contrast, the lower panel of Fig. 9 is an exposure at $4.79 \mathrm{keV}$, i.e. just above the edge. As expected, the effects of penetration are greatly reduced with only a small tail towards lower energies and BL values evident (1). In addition the fraction of escape photons (3) has increased due to the incident photons having sufficient energy to liberate electrons from the Xe $L_{\mathrm{III}}$ shell. A further discussion of these effects can be found in Simons \& de Korte (1989) and Bavdaz et al. (1995a).

\subsection{Gain temperature dependence}

A primary concern is to understand the "gain" of the instrument. This is the relation between the energy of an event and the mean PHA channel at which it is detected. The instrument functional tests were performed over a range of temperatures allowing the temperature dependence of the instrument performance to be investigated. The DU thermistor is used as temperature sensor, since this will be the in-orbit reference.

Each instrument functional test was divided into intervals of constant temperature and for each of these segments the average PHA channel of each calibration source was determined (see Fig. 10). The results from the two calibration sources only differ by a scaling factor which probably arises from a small misalignment of the PMT and gas cell fundamental axes. Both calibration sources show a linear dependence of gain with temperature in the range studied. The best-fit coefficient is $-1.25 \%{ }^{\circ} \mathrm{C}^{-1}$ at a PHA channel of 410 . The scatter in the figure can be 

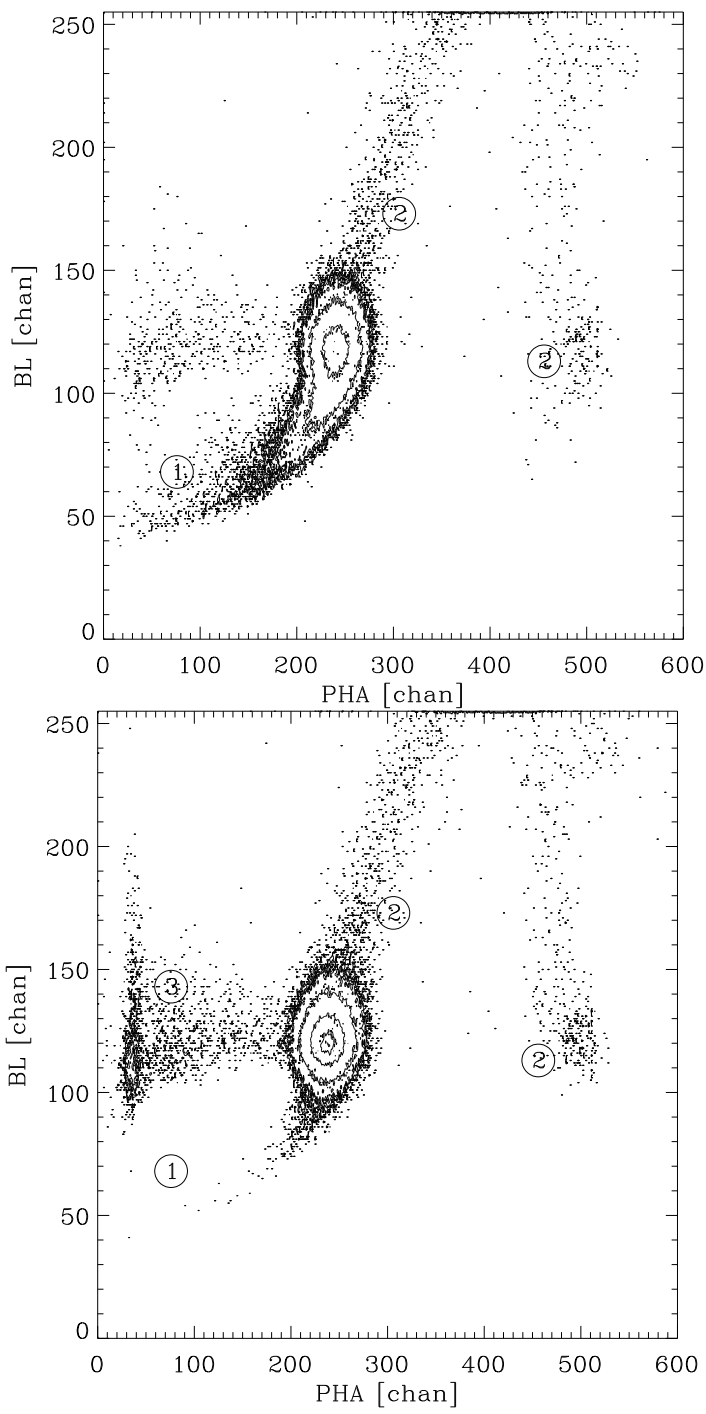

Fig. 9. BL versus PHA channel for $4.77 \mathrm{keV}$ (upper panel) and $4.79 \mathrm{keV}$ photons (lower panel), i.e. just below and above the Xe $L_{\mathrm{III}}$ edge. See the text for a description of the labels

attributed to the $0.4{ }^{\circ} \mathrm{C}$ resolution of the temperature measurement in the region around $25^{\circ} \mathrm{C}$. The same procedure was performed using $\mathrm{Fe} K_{\alpha}$ and $\mathrm{Al} K_{\alpha}$ data from within the FOV to confirm that the temperature dependence is independent of both position and energy. The temperature dependence of the gain can be corrected for by defining a nominal calibration source PHA channel of 410 and normalizing all energy signals to this. If the original energy channel $=$ PHA, then the corrected channel is given by:

$\mathrm{PI}=\mathrm{PHA} \times \frac{410+410}{\mathrm{cal}_{1}+\mathrm{cal}_{2}}$

where $\mathrm{cal}_{1}$ and $\mathrm{cal}_{2}$ are the mean PHA channels of the two calibration sources. Note that the nominal calibration source channel of 410 is that expected at the predicted inorbit LECS temperature of $20{ }^{\circ} \mathrm{C}$.

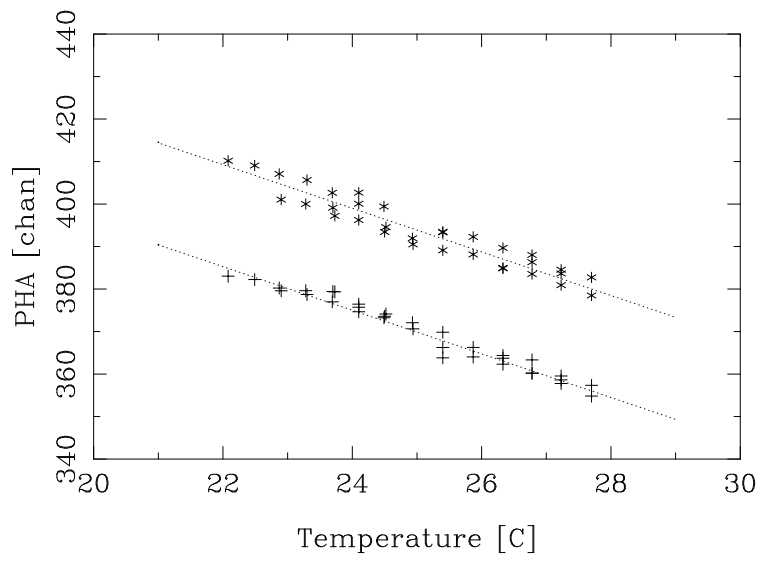

Fig. 10. PHA channel versus temperature for the two ${ }^{55} \mathrm{Fe}$ calibration sources. The dotted lines show the best-fit linear relations

\subsection{Gain position dependence}

Spatial gain maps were obtained by computing the gain at each of the pin-hole locations indicated by circles in Fig. 7. The gain of each image pixel was then estimated by spline interpolation. Temperature effects were removed by dividing the data into intervals of constant temperature and using the average calibration source PHA to normalize the amplitude of each individual X-ray event according to Eq. (5). The peak PI channel was determined by fitting a model consisting of a Gaussian profile plus a constant background to the data. The interpolated gain maps are shown in Fig. 11 for $\mathrm{Fe} K_{\alpha}$ and for $\mathrm{Al} K_{\alpha}$ X-rays. The region of maximum gain is offset slightly from the center of the FOV, probably because of the small misalignment between the PMT and gas cell fundamental. At the edge of the FOV the gain is $\sim 95 \%$ of the maximum. The two gain maps only differ by a scaling factor of 0.238 , consistent with the $\mathrm{Al} / \mathrm{Fe} \mathrm{X}$-ray energy ratio of 0.232 and the energy discontinuities at the Xe $L$ edges.

Additional multi pin-hole measurements were taken at the PANTER facility, where other X-ray energies are available. During this campaign, the pin-hole array was stepped in the $X Y$ plane, parallel to the focal plane, to increase the spatial sampling. These measurements confirm the smooth surfaces and extend the calibration to lower and higher energies. Again, the spatial gain maps are well behaved, differing only by a scaling factor consistent with the $\mathrm{X}$-ray energy ratios and detector gas edge discontinuities.

\subsection{Penetration correction}

Due to the driftless design of the gas cell, X-rays that penetrate into the cell prior to absorption give rise to events with lower PI and BL values than events of the same energy which are detected just beneath the cell entrance window (see Figs. 9 and 12). In order to correct 


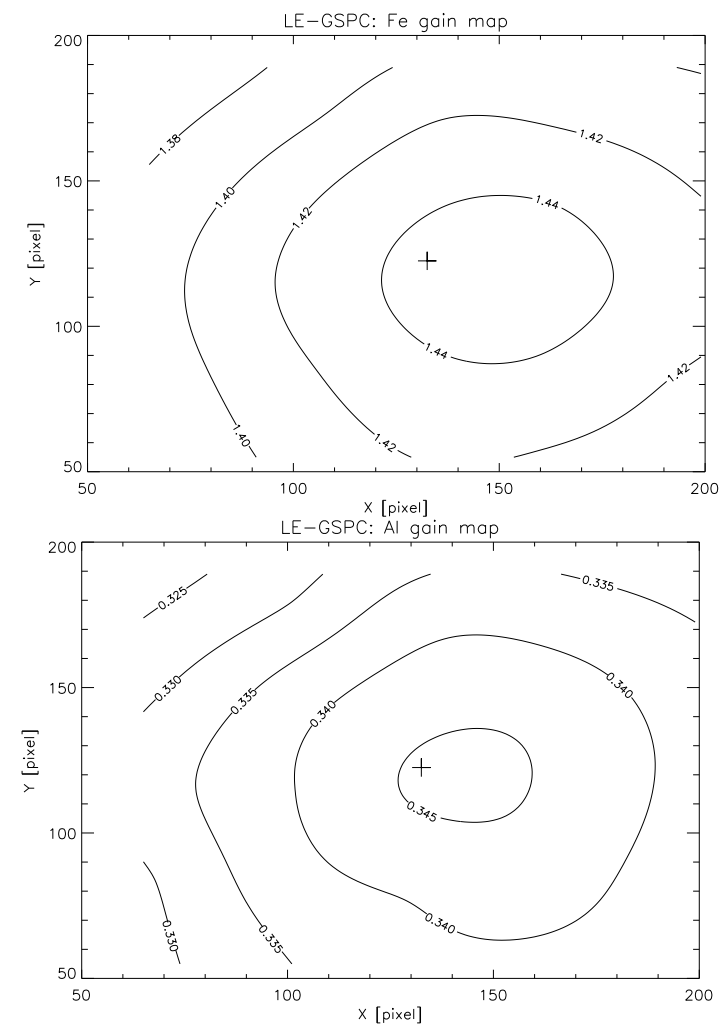

Fig. 11. Gain maps at 1.49 and $6.41 \mathrm{keV}$. The crosses indicates the center of the FOV. The numbers indicate the gain relative to the average of the calibration sources

the observed PI spectra for this effect, data obtained at the PANTER facility have been used to determine the mean PI channel as a function of BL for incident energies $>2.9 \mathrm{keV}$. (Below this energy there is no worthwhile improvement in spectral resolution when this correction is applied). For each line a reference PI channel, $\mathrm{PI}_{\text {ref }}$, was determined. This corresponds approximately to the mean channel of events absorbed directly beneath the cell entrance window. In order to determine $\mathrm{PI}_{\text {ref }}$ for each line, the function described in Eq. (8) together with a constant background were fit to the BL spectra and events with BL values greater than the peak of the distribution selected. A PI spectrum of these selected events was then accumulated and the same function fit in order to account for the remaining small penetration tail. The value of $\mathrm{PI}_{\text {ref }}$ is then given by the best-fit value of $m$. Thus, for a known energy, $E_{\mathrm{c}}$, the observed PI spectrum can be corrected for penetration using:

$\mathrm{PIC}=\mathrm{PI}_{\mathrm{ref}}\left(E_{\mathrm{c}}\right)+\mathrm{PI}-\mathrm{PI}_{m}\left(\mathrm{BL}, E_{\mathrm{c}}\right)$.

Here PIC is the corrected channel and $\mathrm{PI}_{m}$ is the mean PI channel for the observed BL value and incident energy. From an examination of the $\mathrm{PI}_{m}$ distributions it is apparent that the BL resolution of the instrument is insufficient to fully correct for penetration effects in the energy range of interest. This explains the remaining small exponential tail visible in the corrected spectrum in the lower panel of Fig. 12.

During normal astronomical observations there is a further complication that the actual energy of a detected event, $E_{\mathrm{c}}$, is not known a priori and only the probability that an event has a certain incident energy can be estimated. In the LECS data analysis software a value of $E_{c}$ is assigned to each event taking into account this probability. The penetration correction can then be applied using Eq. (6). This lack of a priori knowledge of the incident energy means that e.g. the measured FWHM energy resolution of $9.0 \%$ at $\mathrm{Fe} K_{\alpha}(6.41 \mathrm{keV})$ can only be corrected to $8.6 \%$ using this technique, rather than $8.1 \%$ if the energy of the incident $\mathrm{X}$-rays is known.

\subsection{Energy to channel conversion}

The overall energy gain of the LECS is expected to be linear, with discontinuities at the absorption edges of the detector gas caused by discrete changes in the final ionization state, and hence the amount of energy retained by the $\mathrm{Xe}$ atoms across the absorption edges. In order to measure the overall gain, results from the PANTER measurement campaign were utilized since these covered almost the entire energy range of the instrument. The lines used and their energies (Bearden 1967) are given in Table 2. For each of these lines, the PHA data were first corrected for the overall temperature and position dependence of the gain using Eq. (5) and the mean gain map (see Fig. 11).

The resulting spectra, $\psi(\mathrm{PI})$, can either be represented by a Gaussian or, at energies $\lesssim 1.5 \mathrm{keV}$, a Gaussian plus an exponential low-energy tail. The upper two panels in Fig. 12 show observed line profiles at $0.28 \mathrm{keV}$, where the mean absorption depth is small, and $8.06 \mathrm{keV}$, where it is large (see Fig. 8). The $0.28 \mathrm{keV}$ line profile is well fitted by a Gaussian function, demonstrating the good low-energy performance of the instrument since the loss of significant numbers of electrons would result in a tail towards low energies, which is not evident. The $8.06 \mathrm{keV}$ line shows an exponential tail extending towards lower energies. This results from the penetration of X-rays into the gas cell prior to absorption. The observed line profiles were modeled using a constant background together with the following functions:

- For the B, C, $\mathrm{O}$ and $\mathrm{Cu}_{\mathrm{L}}$ lines in Table 2 where the effects of penetration are not evident:

$$
\psi(\mathrm{PI})=\frac{C_{2}}{\sigma \sqrt{2 \pi}} \exp \left(-\frac{(\mathrm{PI}-m)^{2}}{2 \sigma^{2}}\right) .
$$

- For the other lines in Table 2:

$$
\psi(\mathrm{PI})= \begin{cases}C_{1} \exp (\alpha \mathrm{PI}) & \mathrm{PI} \leq \nu_{0} \\ \frac{C_{2}}{\sigma \sqrt{2 \pi}} \exp \left(-\frac{(\mathrm{PI}-m)^{2}}{2 \sigma^{2}}\right) & \mathrm{PI}>\nu_{0},\end{cases}
$$

where $\nu_{0}$ is the break channel between the two components, $\alpha$ is the e-folding index, $m$ is the peak PI channel 

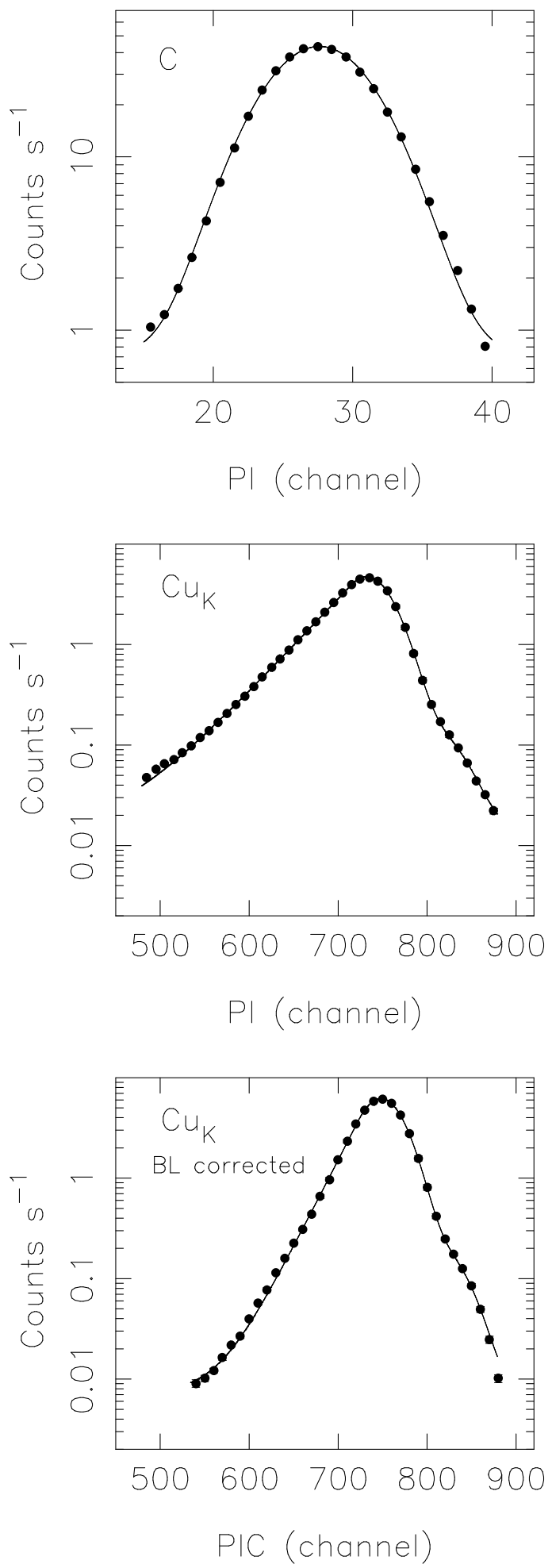

Fig. 12. The response of the LECS to monochromatic radiation at energies of $0.28 \mathrm{keV}$ (upper panel) and $8.06 \mathrm{keV}$ (center panel). The statistical uncertainties are comparable to the symbol size. The "knee" visible in the $\mathrm{Cu}_{\mathrm{K}}$ spectra at channel $\sim 850$ is from $\mathrm{Cu} K_{\beta}$ radiation. The lower panel shows the effect of performing the penetration correction described in Sect. 3.3 on the $\mathrm{Cu}_{\mathrm{K}}$ spectrum assuming an incident energy of $8.06 \mathrm{keV}$ of the Gaussian, $\sigma$ is the width of the Gaussian distribution in standard deviations and $C_{1}$ and $C_{2}$ are the normalizations of the exponential and Gaussian components, respectively. Since the two components connect smoothly at $\nu_{0}$, their first derivatives are equal at this point. (This implies that $\nu_{0}=m-\alpha \sigma^{2}$ ). Some idea of the quality of fits can be obtained from the upper two panels in Fig. 12 where the fitted models are shown as continuous lines. The penetration correction given in Eq. (6) was then applied to the data from all lines with energies $>2.9 \mathrm{keV}$ (below this energy the PI and PIC channels are equivalent). Equations (7) or (8) were then fit to the PIC spectra, as appropriate, to derive the best-fit values of $m$ which are used for the PIC to energy conversion. A linear relation between PIC value and energy was assumed, allowing for a discontinuity at the Xe $L_{\mathrm{III}}$ edge at $4.782 \mathrm{keV}$. The result of this fitting gives:

$\mathrm{PIC}= \begin{cases}1.730+92.478 \cdot E & E \leq 4.782 \mathrm{keV} \\ -1.136+92.478 \cdot E & E>4.782 \mathrm{keV} .\end{cases}$

The discontinuity at the $\mathrm{Xe} L$ edge is $31 \mathrm{eV}$. This value is smaller then the $129 \mathrm{eV}$ measured by Bavdaz et al. (1995a) using a prototype detector at BESSY. This difference probably arises because these authors selected only a small fraction of the available data to minimize penetration effects. Such a technique cannot be usefully applied to astronomical measurements. No evidence for a gain discontinuity at the Xe $M$ edge was found in the PANTER data.

\subsection{Energy resolution}

The energy resolution of the LECS was determined at each of the energies listed in Table 2 using either Eqs. (7) or (8) and corrected for penetration effects using Eq. (6), as appropriate. No a priori knowledge of the line energy was assumed, since this will be the case during astronomical observations. The full width at half-maximum (FWHM) of the PIC energy resolution, $\Delta E$, is related to the width of the Gaussian distribution by $\Delta E=2.354 \sigma$. The LECS energy resolution is given by:

$\frac{\Delta E}{E}=8.84\left(\frac{E}{6 \mathrm{keV}}\right)^{-0.42} \%$

and is shown plotted versus energy in Fig. 13. The deviation from the expected $E^{-0.5}$ relation has been previously noted (Simons \& de Korte 1989) and explained as being due to variations in the footprint of the electron cloud viewed by the PMT.

\subsection{Image linearization}

The coordinate transformation required to translate the RAWX, RAWY pixel coordinates into absolute position on the focal plane were obtained using multi pin-hole 


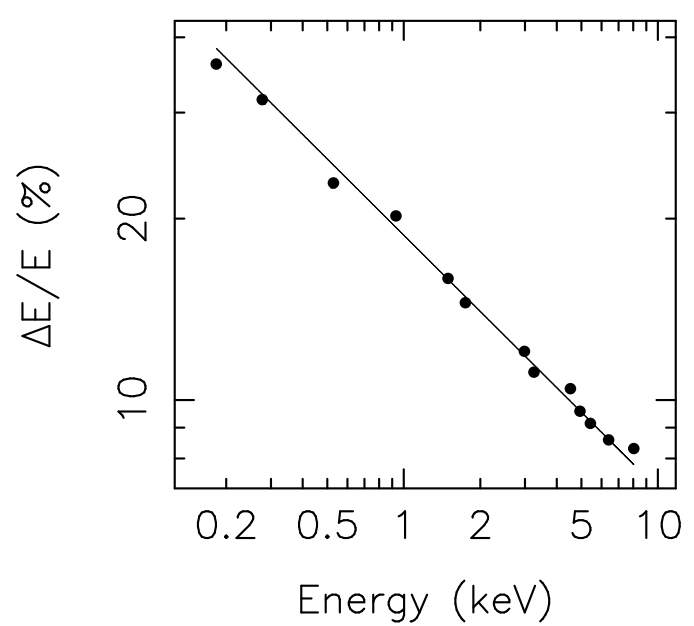

Fig. 13. The LECS penetration corrected energy resolution versus energy. The best-fit relation given in Eq. (10) is shown as a solid line. The uncertainties are smaller than the symbol size

mask (MP) exposures taken at PANTER. Due to the finite source distance of $130 \mathrm{~m}$ the actual distance between consecutive pinholes projected on the focal plane is magnified by $\sim 1 \%$. This effect is taken into consideration in the analysis. The data used for this analysis were obtained using lines of $\mathrm{C}(0.28 \mathrm{keV}), \mathrm{Al}(1.49 \mathrm{keV})$ and $\mathrm{Fe}$ $(6.41 \mathrm{keV})$. For each exposure, the relation between pixel coordinates and pin-hole position, as shown in Fig. 14, was established.

Based on this data, the coordinate transformation required to assign to each RAWX, RAWY pixel its true distance along the $X$ and $Y$ axes from the physical center of the FOV have been determined. A cubic polynomial function has been fit to the data:

$$
\begin{array}{r}
D^{x, y}=a_{0}^{x, y}+a_{1}^{x, y} \cdot p_{x}+a_{2}^{x, y} \cdot p_{y}+a_{3}^{x, y} \cdot p_{x} p_{y}+ \\
a_{4}^{x, y} \cdot p_{x}^{2}+a_{5}^{x, y} \cdot p_{y}^{2}+a_{6}^{x, y} \cdot p_{x}^{2} p_{y}+ \\
a_{7}^{x, y} \cdot p_{x} p_{y}^{2}+a_{8}^{x, y} \cdot p_{x}^{3}+a_{9}^{x, y} \cdot p_{y}^{3} .
\end{array}
$$

Here, $D^{x, y}$ is the distance in $\mathrm{mm}$ from the physical center of the FOV (which is located at pixel coordinates RAWX, RAWY $=131.44,124.12)$ along the $X$ and $Y$ axes, $p_{x}=(\mathrm{RAWX}-136.78), p_{y}=(\mathrm{RAWY}-119.07)$ and $a_{i}^{x, y}$ are the fitted coefficients (one set for each axis). The rms residuals between the data and the fit along both axes are $\sim 60 \mu \mathrm{m}$. This corresponds to about half a pixel and is of the order of the systematics inherent to the experimental set up. From a comparison of the pin-hole images taken at different energies, any energy dependence corresponds to a positional difference of $<100 \mu \mathrm{m}$, except at the edge of the FOV where these may be as large as $200 \mu \mathrm{m}$. The positions in $\mathrm{mm}$ are converted to linearized pixels by the LECS data analysis software assuming a pixel size of $8^{\prime \prime}$.
Table 3. Coefficients required to map pixel coordinates into absolute position on the focal plane

\begin{tabular}{lrr}
\hline & \multicolumn{1}{c}{$x$} & \multicolumn{1}{c}{$y$} \\
\hline$a_{0}$ & $-7.0010^{-1}$ & $-6.8310^{-1}$ \\
$a_{1}$ & $1.2410^{-1}$ & $3.3510^{-3}$ \\
$a_{2}$ & $-5.7110^{-3}$ & $1.2410^{-1}$ \\
$a_{3}$ & $-1.9810^{-5}$ & $7.6210^{-6}$ \\
$a_{4}$ & $4.1610^{-5}$ & $2.2410^{-7}$ \\
$a_{5}$ & $1.4210^{-5}$ & $-2.6510^{-5}$ \\
$a_{6}$ & $8.2410^{-8}$ & $2.0210^{-6}$ \\
$a_{7}$ & $1.9910^{-6}$ & $-4.9210^{-7}$ \\
$a_{8}$ & $1.4510^{-6}$ & $-8.6910^{-8}$ \\
$a_{9}$ & $1.3610^{-7}$ & $2.0410^{-6}$ \\
\hline
\end{tabular}

\subsection{Encircled energy function}

The overall encircled energy function (EEF) of the LECS is a convolution of those of the MU and detector modulated by the obscuration of the entrance window strongback and support grid. Its shape can therefore not be easily parameterized in a similar manner to that of the MECS (Boella et al. 1996a). Measured, azimuthally averaged, onaxis EEFs are shown in Fig. 15. At low energies the EEF is dominated by the contribution of the detector, which is approximately $\propto E^{-0.5}$. This results in the narrowing of the EEF core visible in Fig. 15 with increasing energy. Above $\sim 4 \mathrm{keV}$, the broad scattering wings of the mirror response become important (see Fig. 19). The FWHM of the LECS EEF is given by:

$\mathrm{EEF}=2.1\left(\frac{E}{6 \mathrm{keV}}\right)^{-0.5} \operatorname{arcmin}$

At $6 \mathrm{keV}$ this relation is valid in the central $8^{\prime}$ of the FOV. The range of validity increases with decreasing energy, such that at $0.28 \mathrm{keV}$ it is valid over the entire FOV.

\subsection{Effective area}

The effective area of the LECS is primarily limited at lowenergies by the transmission of the entrance window and at high-energies by the loss in reflectivity of the MU. The predicted on-axis effective area of the LECS is shown in Fig. 16. This figure includes the theoretical mirror efficiency (Sacco 1995, private communication), detector absorption efficiency and plasma grid transmission and the measured entrance and plasma protection window transmissions. A constant geometric obscuration due to the window support structure of $17 \%$ is assumed. The large change in effective area at $0.28 \mathrm{keV}$ is due to the $\mathrm{C} K$ edge. The effect of the $K$-edges of $\mathrm{N}, \mathrm{O}$, and $\mathrm{Al}$, which are all constituents of the windows, are also visible. The structure between $2-4 \mathrm{keV}$ is caused by the M-edges of 

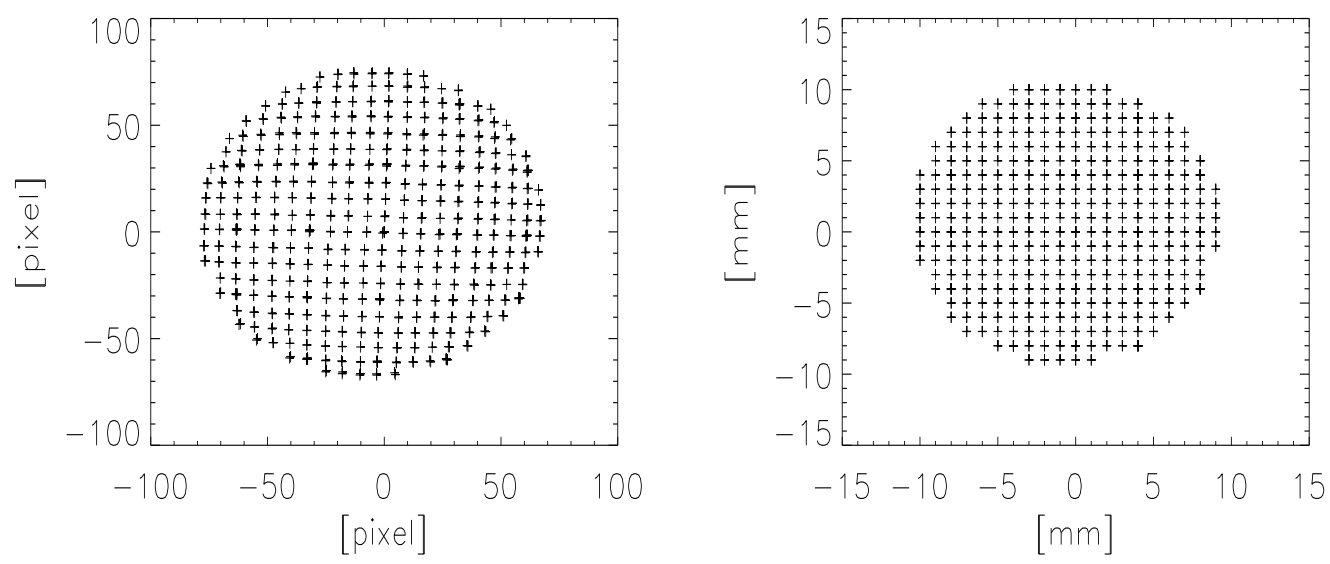

Fig. 14. Correspondence between $p_{x}, p_{y}$ pixels (left panel) and physical space (right panel). Each data point represents the position of one pinhole

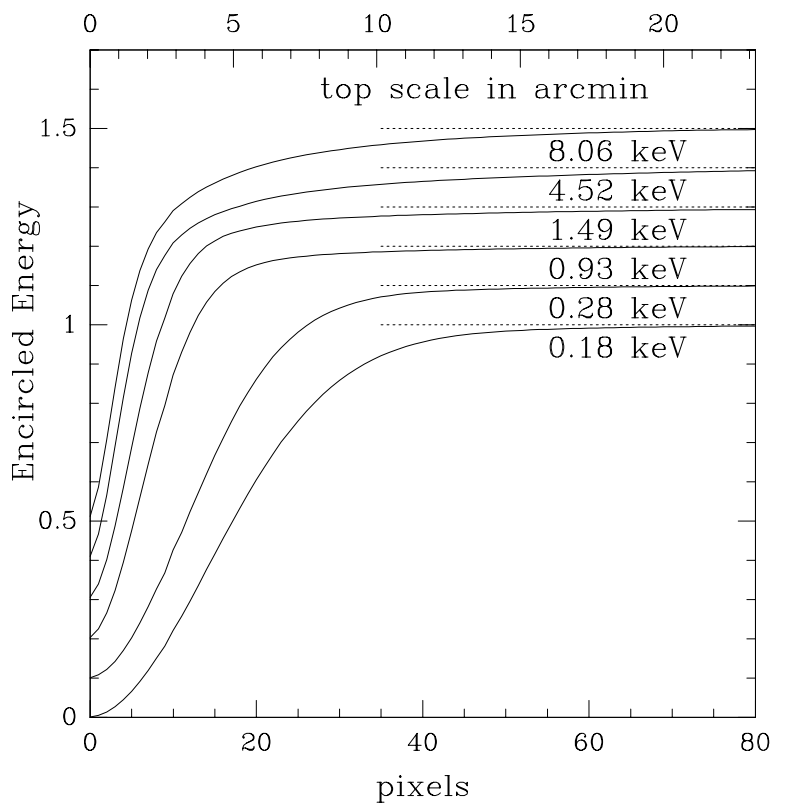

Fig. 15. Measured, azimuthally averaged, on-axis LECS encircled energy functions at various energies. Each plot has been offset from the next by 0.1 for clarity. The dotted lines show the asymptotic values

the Au mirror material. At the edge of the FOV (i.e. an offset angle of $18^{\prime}$ ), the effective area of the LECS is 0.4 times that on-axis.

\subsubsection{Mirror effective area}

In the PANTER configuration the effective mirror collecting area is reduced to $97.3 \mathrm{~cm}^{2}$, including obscuration by the mirror spider (Conti et al. 1994). The MU effective

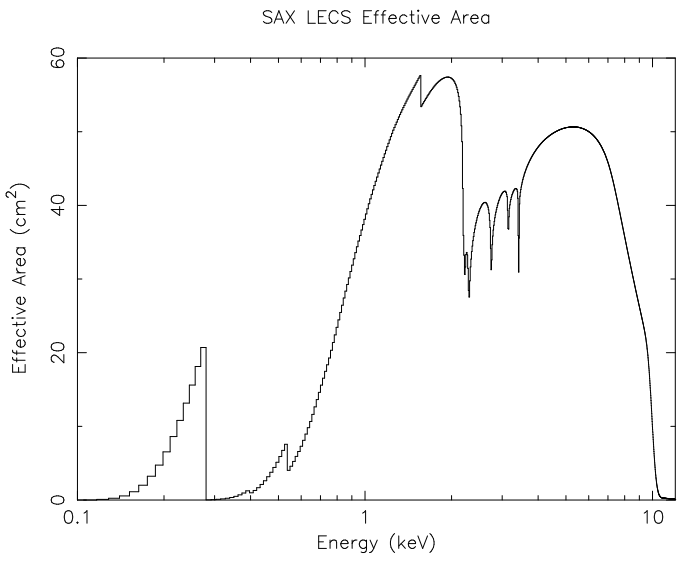

Fig. 16. Predicted LECS on-axis effective area

area was measured at each of the energies listed in Table 2 (except that the measurements for the two $\mathrm{Ag}$ and $\mathrm{Ti}$ lines were combined, and an additional P $(2.01 \mathrm{keV})$ measurement was included). The MU effective area was derived by comparing the count rate with the MU in place to that during a flat-field exposure (i.e. with the MU removed). The derived values were corrected for the effects of strongback and fine grid obscuration and deadtime and are shown plotted in Fig. 17. This figure also shows the theoretical MU response derived from ray-tracing simulations of a source at the same distance as that at PANTER. The agreement between measurement and theory is good except at low energies (the $\mathrm{B}$ and $\mathrm{C}$ measurements at 0.18 and $0.28 \mathrm{keV}$ ) where the measured areas are a factor $\sim 1.5$ too high. Such an effect may be related to the location of the LECS in the PANTER chamber and the complicated behavior of the MU to off-axis X-rays. In particular, 
$\mathrm{X}$-rays from within a $2^{\circ}$ offset angle which are reflected by either one of the first or second mirror cones only, can reach the focal plane. In addition, for particular offset angles, X-rays can reach the focal plane without reflection (Conti et al. 1994). Thus, it is possible that low-energy $\mathrm{X}$-rays scattered off the chamber walls during the mirror exposures enter the detector, resulting in the anomalous area measurements. An additional effect may be due to molecular contamination of the mirror surfaces (e.g., Elsner et al. 1994). However, there is no totally convincing explanation for this phenomenon and we await the results of in-flight measurements to verify the low-energy LECS effective area.

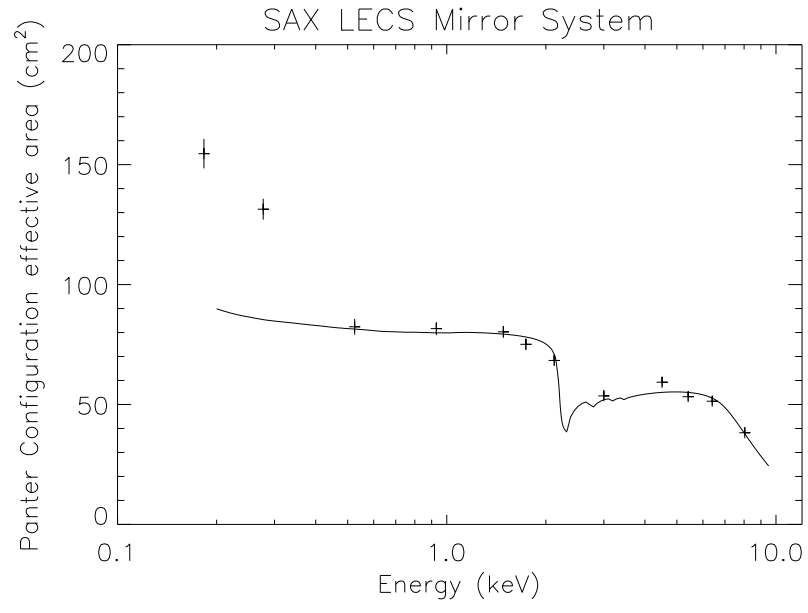

Fig. 17. Measured and theoretical (solid line) on-axis MU effective area versus energy in the PANTER configuration

\subsubsection{Strongback and grid obscuration}

The construction of the strongback and grid are shown in Figs. 4 and 5, respectively. The strongback and grid together obscure an average of $17 \%$ of normally incident $\mathrm{X}$-rays. However, this obscuration depends on both position in the FOV and X-ray energy in a complex way. The energy dependence arises because:

- The width of the concentrator EEF is energy dependent, being broader at higher energies (see Fig. 19). This means that the area of support structure that is illuminated depends on energy.

- The finite strongback height casts a shadow onto the grid for incident X-rays that do not arrive normally. Since the average $\mathrm{X}$-ray incidence angle is energy dependent, this effect is also energy dependent.

The effect of strongback and fine grid obscuration is illustrated in Fig. 18 which shows the LECS counting rate at $0.28 \mathrm{keV}$ during a $3.9 \mathrm{~mm}$ long scan of the central region

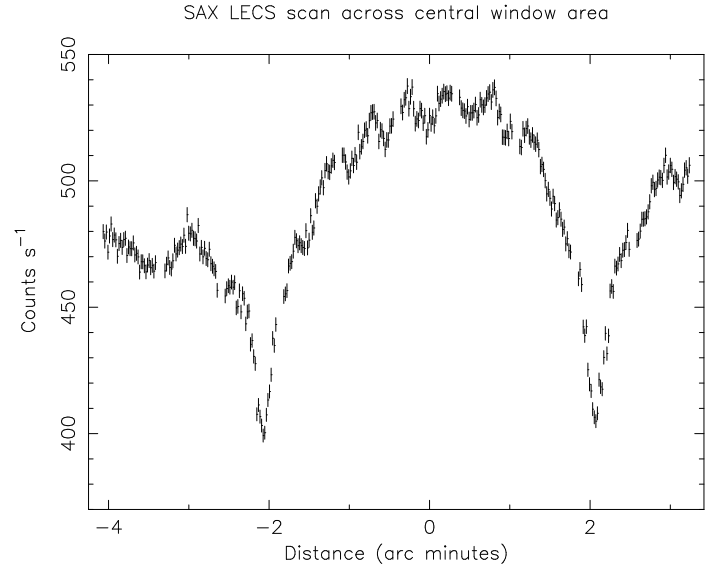

Fig. 18. A scan across the central part of the LECS window centered on $0.28 \mathrm{keV}$ showing the effects of strongback and fine grid obscuration. The distance scale indicates the approximate angular distance from the center of the FOV

of the detector in a direction parallel to the $Y$ axis, using a constant intensity X-ray beam. The two large $(\sim 25 \%)$ decreases in count rate delineate the $2.2 \mathrm{~mm}$ central strongback square and are caused by the strongback ribs. A smaller amplitude $(\sim 5 \%)$ modulation due to the fine grid is also visible. The overall trend of increasing count rate with distance along the $Y$ axis is probably due to a small misalignment between the mirror and detector units.

These obscuration effects are treated by means of a Monte-Carlo simulation in the LECS data analysis system. The first stage of this process is a ray-tracing model of the mirror system. This is based on the commercial ray tracing software package, IRT, from Parsec Technology Inc. which has been "tuned" to give good agreement with the mirror EEF's obtained at the PANTER facility using the flight MU and the ROSAT Position Sensitive Proportional Counter (PSPC) as a focal plane detector. The spatial resolution of the PSPC is much better than that of the LECS, and is sufficient to allow the intrinsic shape of the MU EEF to be reliably determined. Figure 19 shows the good agreement between observed MU EEFs and those predicted by the ray-tracing model.

A geometric model of the support structure and the fine grid is then used to determine the fate of an X-ray with given energy and arrival direction. X-rays which exit the MU can reach the entrance window undisturbed, or be absorbed in the fine grid, or interact with the strongback. In the latter case, the photon can be either absorbed or reflected, with a probability that depends on the energy and the angle of incidence with the strongback. Reflected photons may be absorbed by the fine grid. Using the processes described above, it is possible to generate the EEF of the concentrators for any given energy and position in the FOV and to propagate this through the support 
structure. The effect of this obscuration on the analysis of both extended and variable sources will be evaluated during the Science Verification Phase of the mission.

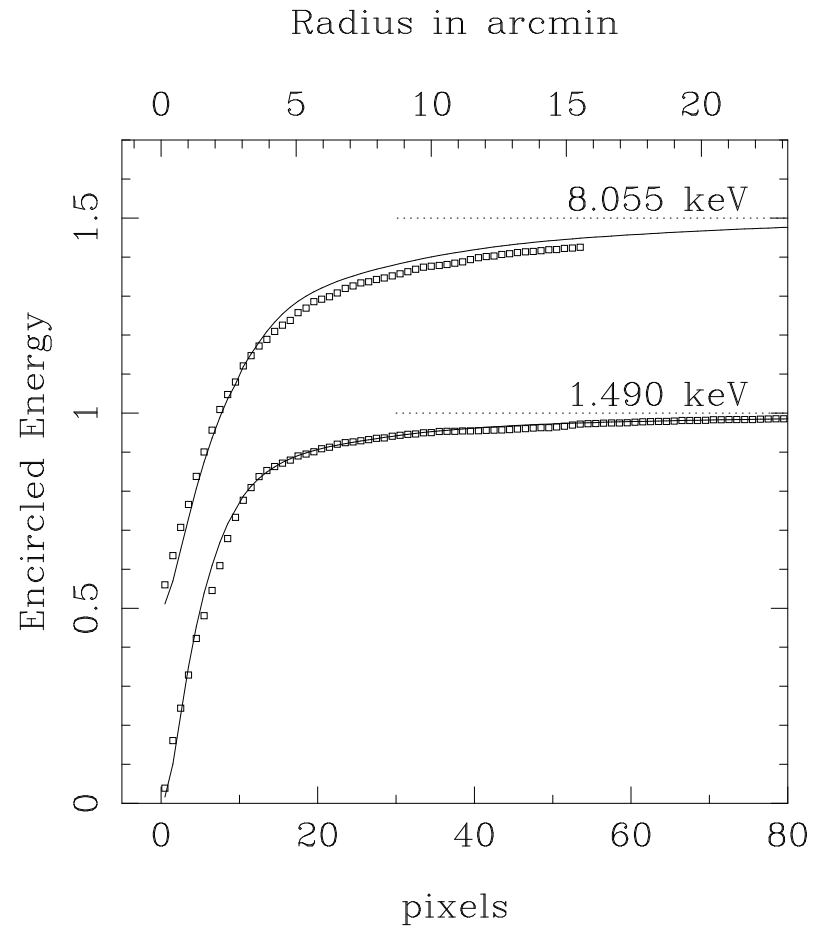

Fig. 19. Azimuthally averaged mirror unit EEFs at two energies obtained by ray-tracing (squares) and measured using the ROSAT PSPC at two energies (solid lines). The plots have been offset by 0.5 units for clarity. The dotted lines show the asymptotic values

\subsubsection{Entrance window X-ray transmission}

The primary goal of the LECS is to perform low-energy spectroscopy. Since the low-energy response of the detector is primarily defined by the transmission of the entrance window, a well calibrated window transmission is vital. With this in mind, great care was taken to determine the window transmission paying particular attention to variations near the edges of the constituent materials and to the position dependence of the overall transmission.

Measurements using the available lines at PANTER (Table 2) are inadequate for this purpose, and instead a thorough investigative campaign was performed at BESSY. The PTB SX-700 plane grating monochromator beamline was used which provided energy resolution, $E / \Delta E$, of between 5000 at $0.06 \mathrm{keV}$ to 400 at $1.8 \mathrm{keV}$, scaling as $E^{-0.5}$. Since the entrance window is highly transparent at energies $\lesssim 2 \mathrm{keV}$, measurements were only made in the energy range 0.1 to $1.8 \mathrm{keV}$. Transmissions at 500 energy values were measured at five positions in the central window area. Energy steps as small as $0.2 \mathrm{eV}$ were used around the absorption edges of the constituents. These measurements were complemented by a series of measurements in the center of each of the 8 by 8 fine grid positions in the central 3 by 3 central strongback squares (see Fig. 4). Between 18 and 30 energies were used for these scans. The $99 \%$ flux width of the beam was $\sim 180 \mu \mathrm{m}$, smaller than the fine grid mesh size. This allowed the transmission of the window to be measured separately from the obscuration caused by the support structure. The absolute errors on the transmission are $\sim 1 \%$ and the variations in transmission with position $<3 \%$. A detailed discussion of these measurements is to be found in Bavdaz et al. (1994, 1995b).

The X-ray cross sections used by Bavdaz et al. (1994) were derived from tables in Veigel et al. (1971). Another, more recent, set of coefficients has been published by Henke et al. (1993). They differ from the older ones mainly around the absorption edges and are used for the LECS calibration. The usual approach to modeling X-ray transmission, $T$, through an absorbing layer is to use:

$T(x, E)=\exp (-\mu \rho x)$

where $\rho$ is the density, $x$ the thickness and $\mu$ the mass absorption coefficient, which for compounds is given by:

$\mu=\sum_{i} \mu_{i} f_{i}$

where $\mu_{i}$ is the mass absorption coefficient of element $i$ and $f_{i}$ its fractional weight. By defining $a_{i}$ as the area density of element $i$, we can write:

$\sum_{i} \mu_{i} f_{i} \rho x=\sum_{i} \mu_{i} \rho_{i} x=\sum_{i} \mu_{i} a_{i}$

Using the measurements at different energies, gives the area densities required to describe the X-ray transmission properties of the window. Unfortunately the chemical state and thickness of the absorber change the edge absorption characteristics e.g., Bearden \& Burr (1967) and Owens et al. (1996) and Eq. (14) is only a good approximation away from the edges. As an example, Fig. 20 shows the actual transmission around the $\mathrm{O} \mathrm{K}$ edge compared to the Veigel et al. (1971) (solid line) and Henke et al. (1993) mass absorption coefficients (dashed line).

In order to precisely model the LECS window transmission, the following empirical function has been used:

$T=\exp \left(-\sum_{i} \mu_{i} a_{i}\right) \times T_{\mathrm{c}}$

where the correction factor, $T_{\mathrm{c}}$, is defined as the ratio of the measured data to that predicted using the Henke et al. (1993) coefficients outside the edge regions:

$T_{\mathrm{c}}=T_{\text {data }} / \exp \left(-\sum_{i} \mu_{i} a_{i}\right)$ 
The correction factor is derived from the average of the five high resolution energy scans and is plotted in Fig. 21. As expected, it has a value close to one (i.e. no correction) away from the edges of constituent materials and above $1.8 \mathrm{keV}$.

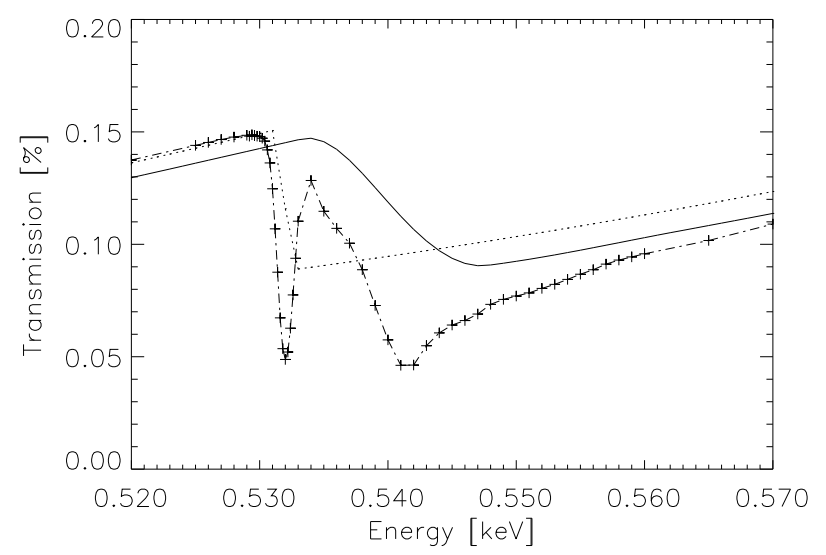

Fig. 20. Entrance window transmission around the O K edge. Predictions using theVeigel et al. (1971) and theHenke et al. (1993) mass absorption coefficients are shown as solid and dashed lines, respectively. The measured data are shown as crosses

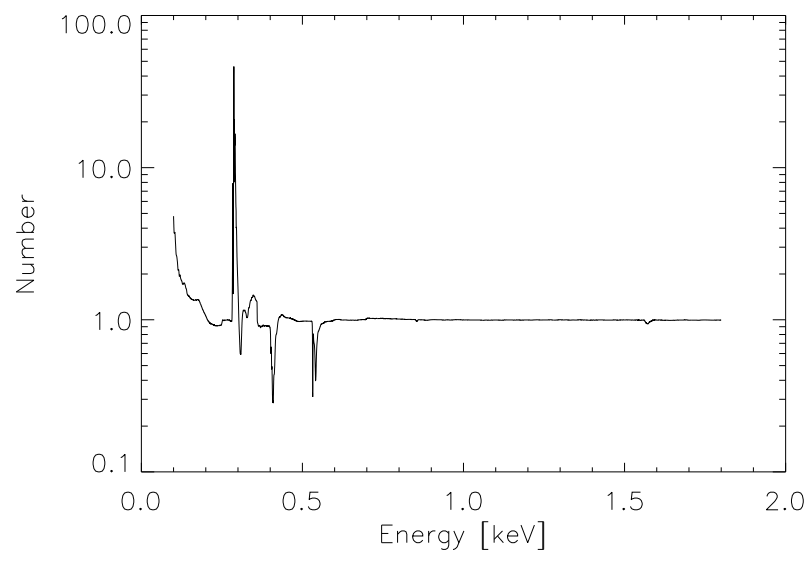

Fig. 21. The correction factor used to model the transmission of the entrance window around the absorption edges

The area densities for each of the window constituents were fit at each position by minimizing the parameter, $Q$ :

$$
\begin{gathered}
Q \equiv \sum_{j}\left(T_{\mathrm{data}}^{j}-T_{\exp }^{j}\right)^{2} \\
T_{\exp }^{j}=\exp \left(-\sum_{k=1}^{5} \mu_{k}^{j} a_{k}\right)
\end{gathered}
$$

where the index " $j$ " denotes the energy dependence and " $k$ " the window constituents of $\mathrm{H}, \mathrm{C}, \mathrm{N}, \mathrm{O}$, and $\mathrm{Al}$.
Energy regions close to the absorption edges were excluded. Table 4 summarizes the theoretical composition, based on the design shown in Fig. 3. Figure 22 shows the measured transmission of the entrance window and compares it with a $10 \mu \mathrm{m}$ thick Be window as used on the ASCA GSPC (Tanaka et al. 1994). The derived area densities are given in Table 5 . The fitted curve matches the data well, but is not superposed for clarity. The complex structure near the edges is clearly visible.

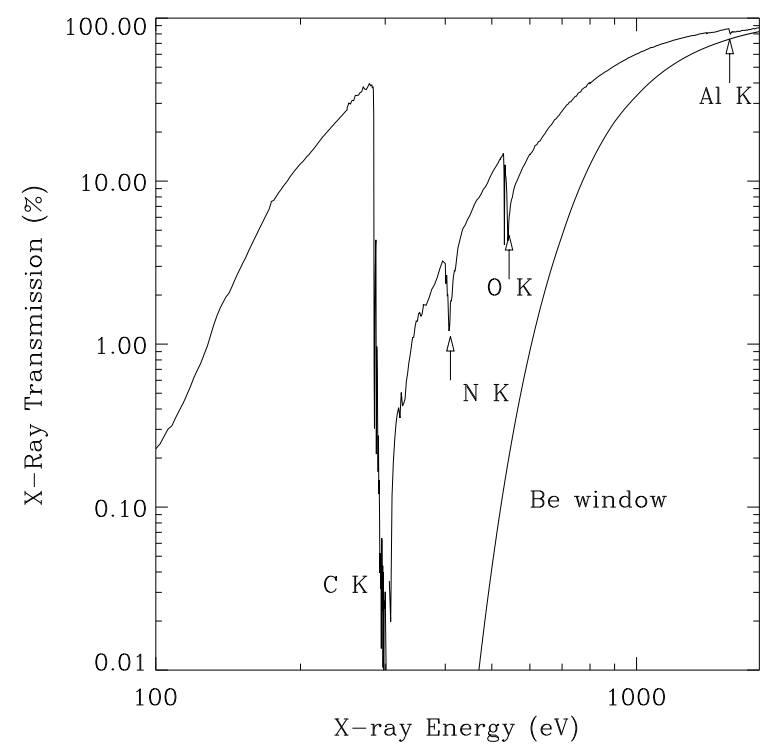

Fig. 22. The measured transmission of the LECS entrance window at one position. Absorption edges of the constituent materials are indicated. The theoretical transmission of a $10 \mu \mathrm{m}$ Be window is also shown for comparison

Table 4. Nominal entrance window composition

\begin{tabular}{lll}
\hline Material & $\begin{array}{l}\text { Area density } \\
\left(\mu \mathrm{g} \mathrm{cm}^{-2}\right)\end{array}$ & $\begin{array}{l}\text { Thickness } \\
(\mu \mathrm{m})\end{array}$ \\
\hline Polyimide & 175 & 1.25 \\
AlN & 11.4 & 0.035 \\
$\mathrm{Al}$ & 11.9 & 0.044 \\
\hline
\end{tabular}

\subsubsection{Plasma protection window X-ray transmission}

The transmission of the plasma protection window was also measured at BESSY. A high resolution energy scan (500 energy measurements between 0.1 and $1.8 \mathrm{keV}$ ) at the center of the window and a high position resolution 
Table 5. Measured entrance window composition

\begin{tabular}{|c|c|c|}
\hline \multirow[t]{2}{*}{ Element } & \multicolumn{2}{|c|}{$\begin{array}{c}\text { Area density } \\
\left(\mu \mathrm{g} \mathrm{cm}^{-2}\right)\end{array}$} \\
\hline & nominal & measured \\
\hline $\mathrm{H}$ & 4.8 & 4.9 \\
\hline $\mathrm{C}$ & 126 & 130 \\
\hline $\mathrm{N}$ & 17.3 & 17.1 \\
\hline $\mathrm{O}$ & 31 & 38 \\
\hline $\mathrm{Al}$ & 19.4 & 12.8 \\
\hline
\end{tabular}

scan at 3 energies $(0.27,0.90$ and $1.55 \mathrm{keV})$ were performed. The scan revealed $<1 \% \mathrm{rms}$ spatial variations in transmission. Since this window is located $5 \mathrm{~cm}$ above the focal plane, any small transmission variations will be smoothed out and we therefore assume a uniform absorption with position. The absorption of the window has been modeled in a similar manner to that of the entrance window, except that the effect of the support grid is included. The resulting function is plotted in Fig. 23.

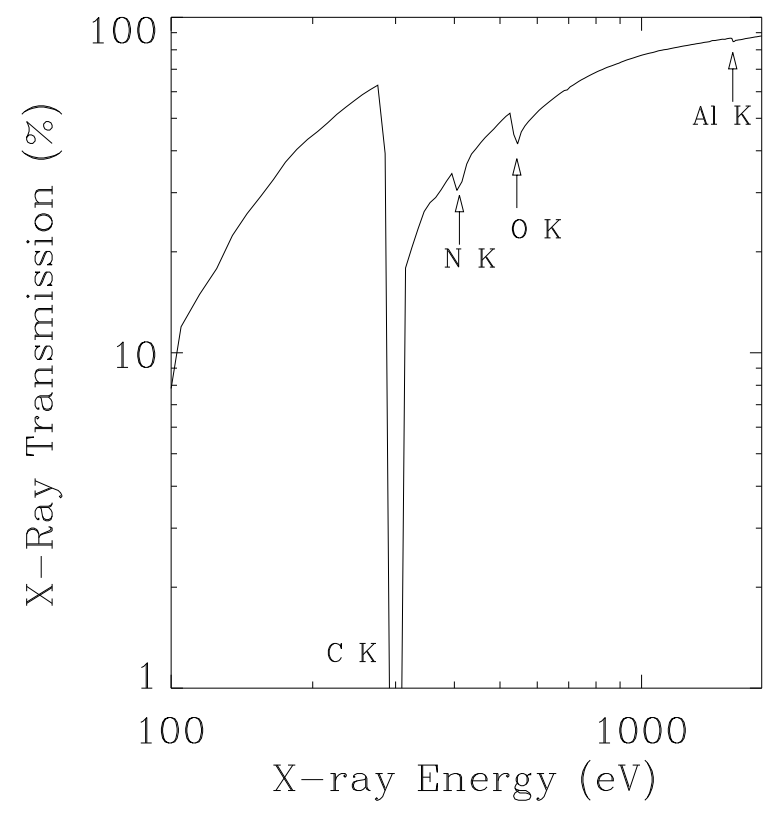

Fig. 23. The measured transmission of the plasma protection window and strongback. Absorption edges of the constituent materials are indicated

\subsubsection{Detector absorption efficiency}

Nearly all incident X-rays with energies in the range $0.1-10 \mathrm{keV}$ that pass through the entrance window are absorbed in the $5 \mathrm{~cm}$ deep LECS gas cell. The absorption efficiency decreases slightly at the upper end of the energy range, being 0.996 at $10 \mathrm{keV}$. Following photo-electric absorption an excited ion may relax by the emission of an Auger electron or a fluorescent photon. The probability that a fluorescent photon will escape from the detection volume is geometry dependent, and has been measured for the LECS using data obtained at BESSY and PANTER. The values given in Table 6 have been adopted for use in the LECS calibration and show that $1.68 \%$ of incident $\mathrm{X}$-rays with energies just above the Xe $L_{\mathrm{I}}$ edge produce fluorescent X-rays which are lost from the instrument, resulting in escape peaks. This figure is less than the Xe $L$ shell fluorescent yield of $\sim 8 \%$ due to the probability that an emitted fluorescent photon is absorbed within the detector. Table 6 gives the escape fractions for energies between the Xe $L_{\mathrm{III}}$ and Xe $L_{\mathrm{I}}$ edges. Above the Xe $L_{\mathrm{I}}$ edge the escape fraction is assumed to decrease linearly with energy, falling to $1.12 \%$ at $8.06 \mathrm{keV}$.

Table 6. LECS escape line data

\begin{tabular}{|c|c|c|c|c|}
\hline \multicolumn{2}{|c|}{ Edge } & \multicolumn{2}{|c|}{ Fluorescent line } & \multirow{2}{*}{$\begin{array}{c}\text { Probability } \\
(\%)\end{array}$} \\
\hline Name & $\begin{array}{c}\text { Energy }^{a} \\
(\mathrm{keV})\end{array}$ & Name & $\begin{array}{c}\text { Energy }^{b} \\
(\mathrm{keV})\end{array}$ & \\
\hline$L_{\mathrm{I}}$ & 5.453 & $L_{\beta_{3}}$ & 4.512 & 0.15 \\
\hline \multirow[t]{2}{*}{$L_{\mathrm{II}}$} & 5.104 & $L_{\gamma_{1}}$ & 5.036 & 0.02 \\
\hline & & $L_{\beta_{1}}$ & 4.422 & 0.46 \\
\hline \multirow[t]{2}{*}{$L_{\text {III }}$} & 4.782 & $L_{\beta_{2}}$ & 4.720 & 0.12 \\
\hline & & $L_{\alpha_{1}}$ & 4.111 & 0.93 \\
\hline
\end{tabular}

${ }^{a}$ Bearden \& Burr (1967), ${ }^{b}$ Bearden (1967).

\subsection{Background counting rate}

The VETO and BL signals can be used to minimize the residual background counting rate seen in the LECS. The definition of these signals is given in Sects. 2.2 and 2.3. Briefly, the VETO signal is a measure of the fraction of the total light collected by the central anode of the PMT (see Fig. 6) and, in general, an on-axis event will have a higher value than an off-axis or extended event. The BL signal is a measure of the duration of scintillation of an event and will, on average, be shorter for events that penetrate into the detector prior to absorption.

Figure 24 shows BL plotted against VETO for on-axis $\mathrm{B}(0.18 \mathrm{keV})$ and $\mathrm{Cu}_{\mathrm{K}}(8.06 \mathrm{keV})$ events. Events falling outside the FOV are excluded. The events with VETO values of $\sim 20$ result from multiple calibration source events being detected during a single EU sampling window. The effects of penetration are clearly seen in the $\mathrm{Cu}_{\mathrm{K}}$ image, where a tail towards lower BL and higher VETO values is evident. This change in VETO value is caused by the 

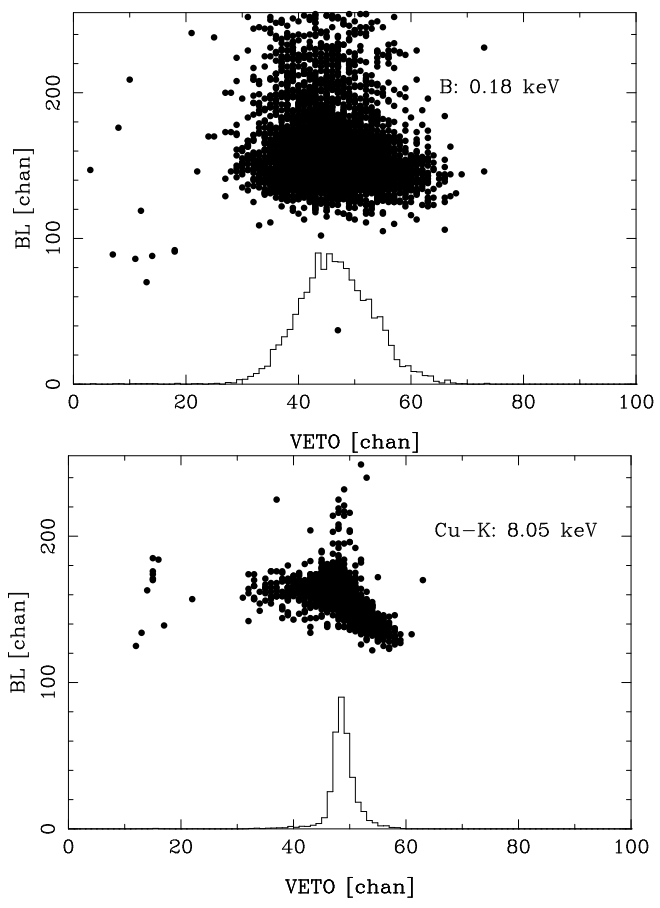

Fig. 24. BL plotted against VETO at two energies

increased PMT viewing angle of penetrating events which are absorbed closer to the central anode (see Fig. 6 and Eq. (4)). At $0.18 \mathrm{keV}$, because of low signal strength, the BL signal distribution is significantly broadened by the EU circuitry. In addition, at low energies there is an unexpectantly large number of events with high BL values. The magnitude of this effect appears to be inversely correlated with mean absorption depth. It is possible that these events are absorbed very close to the entrance window where the electric field is non-uniform due to the undulating window surface. Figure 25 shows an optical image of part of an entrance window foil and illustrates the irregular nature of the foil in each of the 250 by $250 \mu \mathrm{m}$ squares which correspond to the gridlets of the window support grid (see Fig. 5).

Figure 26 shows the allowed range of $\mathrm{BL}$ plotted against PI energy channel for a $90 \% \mathrm{X}$-ray acceptance as a hatched area. The broadening of the BL distribution at low-energies discussed above is visible, as is the effect of change in mean absorption depth around the Xe L edge at a PI channel of $\sim 400$. This figure has been obtained using PANTER data for the lines shown in Table 2 (except that the results for the $\operatorname{Ag} L_{\alpha}$ and $L_{\beta}$ and Ti $K_{\alpha}$ and $K_{\beta}$ lines have been combined) and by making a series of "box cuts" centered on the calibration energies.

The LECS background counting rate within the FOV has been estimated using a $3.610^{5} \mathrm{~s}$ exposure taken in SSD's X-ray beam. A VETO acceptance interval of 30 to 65 combined with $\mathrm{BL}$ cuts of the type described above were applied to the data to give count rates

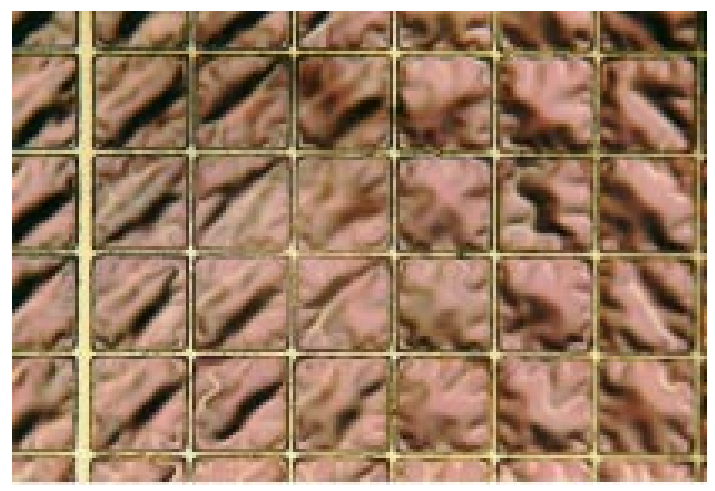

Fig. 25. An optical image of part of an entrance window foil showing the irregular nature of the foil in each of 250 by $250 \mu \mathrm{m}$ gridlets. The lightly colored thin lines show the location of part of the window support grid. Part of a strongback rib is located over the thicker line

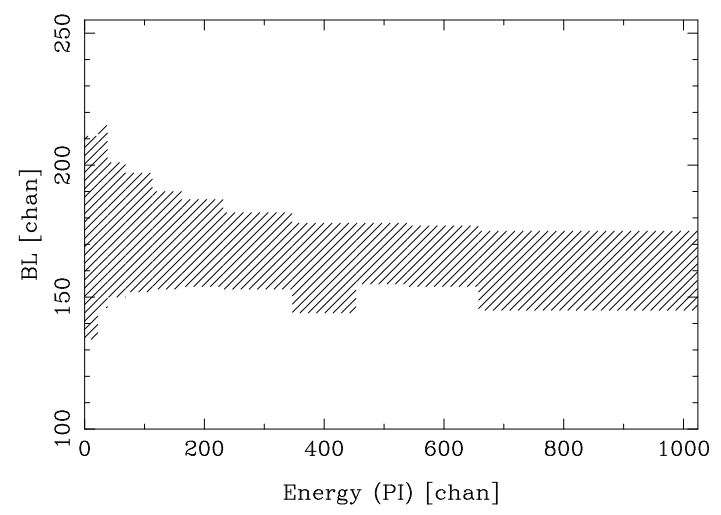

Fig. 26. The allowed BL range for $90 \% \mathrm{X}$-ray acceptance efficiency shown as a hatched region

of $1.610^{-3} \mathrm{~cm}^{-2} \mathrm{~s}^{-1} \mathrm{keV}^{-1}$ for $90 \%$ X-ray acceptance and $1.810^{-3} \mathrm{~cm}^{-2} \mathrm{~s}^{-1} \mathrm{keV}^{-1}$ for $99 \% \mathrm{X}$-ray acceptance. (Note that the $\mathrm{cm}^{-2}$ in the count rate units refers to the window area). Figure 27 shows the effect of these selections. The increase in counts observed in the incident spectrum around PI channel 600 is primarily due to event pile up from the calibration sources. As Fig. 27 illustrates, these events are efficiently removed by the VETO selection. At the lowest PI channels there is an increase in detected event rate which is probably due to electronic noise. The EU analog thresholds were modified following the PANTER calibration and this effect is not expected to occur in space.

\section{Data analysis software}

The BeppoSAX Operations Control Center will not perform any processing of the scientific data which means that observers will receive a time-ordered telemetry stream separated into event and housekeeping data for each interval of contiguous instrument configuration and 


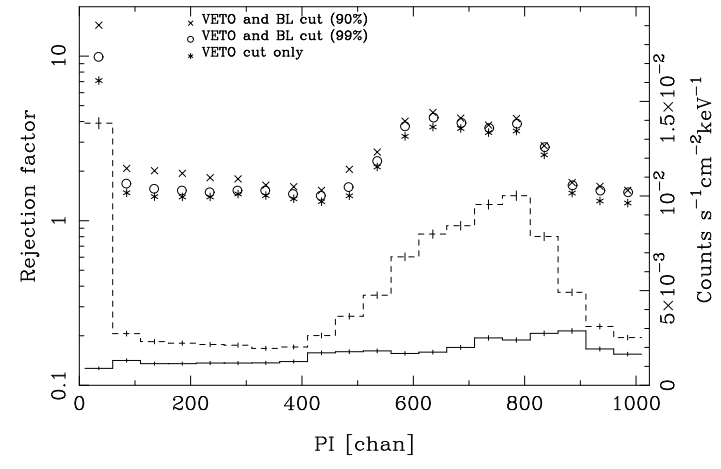

Fig. 27. The effect of VETO and BL selection on the laboratory background spectrum within the FOV. The observed spectrum is shown as a dashed histogram and the spectrum remaining following selection as a solid histogram. $1 \sigma$ uncertainties are indicated. The count rate scale is given by the right ordinate. The efficacy of each of the selection techniques is given by the left ordinate. See the text for a description of the spectra

spacecraft pointing. In addition, auxiliary data such as the geomagnetic cutoff rigidity and the angle of the instrument's pointing axis with respect to the Earth's limb are not present on BeppoSAX data tapes and have to be calculated if they are to be used for data screening. Since the event files cannot be directly read into any of the major data reduction packages currently in use by the astronomical community, a dedicated LECS software package has been developed by SSD which will be made available to observers through the BeppoSAX operations center in Rome.

The LECS software allows the following steps to be performed:

- Conversion of the event and housekeeping telemetry files into a set of FITS format files, following the conventions which are becoming a de facto standard in the astronomical community.

- Generation of auxiliary quantities data.

- Monitoring of relevant housekeeping parameters.

- Determination of the gain of the two calibration sources.

- Selection of the "good" events and time intervals and rejection of background events, based on criteria such as VETO and BL ranges, position in the FOV, Earth limb angle, etc.

- Conversion from raw PHA channels to PI channels for each event using the gain of the two calibration sources as input (see Sect. 3.1).

- Linearization of event positions using the calibration data (see Sect. 3.6) and conversion to sky coordinates.

- Extraction of images, spectra and light curves for subsequent scientific analysis.
- Generation of the detector response matrix for the source being analyzed, using as input the extracted spectrum and relevant calibration data.

Two options are available in the LECS data analysis system to correct the PI data for the effects of penetration. The first is to leave the observed spectra uncorrected i.e. with non-Gaussian tails at energies where the penetration depth is significant (see Fig. 12) and to generate the appropriate detector response matrix incorporating this effect. The second option is to correct the PI data based on event BL as described in Sect. 3.3 and so obtain the best possible spectral resolution from the instrument.

The LECS calibration data are stored in a database which is accessed at run time by the relevant modules. This makes updates of the calibration simpler, as no modification or re-compilation of the software is necessary, and only the relevant calibration files have to be updated in the database.

Acknowledgements. We thank M. Adriaens, S. Andersson, E. Dutruel and T. Paulsen for their support during the development and testing of the LECS. H. Brauninger, W. Burkert, C. Erd, R. Much and A. Poelaert are thanked for their support during calibrations. B. Sacco and G. Conti are thanked for providing information on the calibration of the BeppoSAX mirrors prior to publication and A. Smith is thanked for discussions. E. Kuulkers acknowledges an ESA Research Fellowship. Finally, we thank the ASI Space Segment project manager, R.C. Butler, for his tireless efforts on behalf of BeppoSAX.

\section{References}

Anger H.O., 1958, Rev. Sci. Instr. 29, 27

Bavdaz M., Martin D.D., Peacock A., 1995a, SPIE 2517, 465

Bavdaz M., Peacock A., Fuchs D., et al., 1995b, Rev. Sci. Instrum. 66, 2570

Bavdaz M., Peacock A., Parmar A.N., et al., 1994, Nucl. Inst. Meth. A 345, 549

Bearden J.A., 1967, Rev. Mod. Phys. 39, 78

Bearden J.A., Burr A.F., 1967, Rev. Mod. Phys. 39, 125

Bignami G.F., Villa G.E., Boella G., et al., 1990, SPIE 1344, 144

Boella G., Chiappetti L., Conti G., et al., 1996a (this volume)

Boella G., Butler R.C., Perola G.C., et al., 1996b (this volume)

Butler R.C., Scarsi L., 1990, SPIE 1344, 46

Citterio O., Conti G., Mattaini E., Sacco B., Santambrogio E., 1985, SPIE 597, 102

Conti G., Mattaini E., Santambrogio E.B., et al., 1994, SPIE 2279, 101

Davelaar J., Manzo G., Peacock A., Taylor B.G., Bleeker J.A.M., 1980, IEEE Trans. Nucl. Sci. NS-27, 196

Elsner R.F., Joy M., O'Dell S.L., Ramsey B.D., Weisskopf M.C., 1994, SPIE 2279, 12

Henke B.L., Gullikson E.M., Davis J.C., 1993, Atom. Data Nucl. Data Tab. 54, 1

Holland A.D., Abbey A., Lumb D.H., McCarthy K., 1990, SPIE 1344, 386

Inoue H., Koyama K., Matsuoka M., Ohashi T., Tanaka Y., 1978, Nucl. Instr. Meth. A 157, 295 
Owens A., Denby M., Wells A., et al., 1996, ApJ (submitted) Peacock A., Andresen, R.D., Leimann E.A., et al., 1980, Nucl. Instr. Meth. A 169, 613

Perola G.C., 1983, The Scientific Objectives of the BeppoSAX mission. In: Perola G.C., Salvati M. (eds.) Proceedings of the Workshop on Non-thermal and Very High Temperature Phenomena in X-ray Astronomy, Rome, p. 175

Scarsi L., 1993, A\&AS 97, 371

Simons D.G., de Korte P.A.J., 1989, Nucl. Inst. Methods A 277,642

Simons D.G., de Korte P.A.J., Peacock A., Bleeker J.A.M., 1985, SPIE 597, 190

Spada G.F.L., 1983, BeppoSAX Scientific Instrumentation. In: Perola G.C., Salvati M. (eds.) Proceedings of the Workshop on Non-thermal and Very High Temperature Phenomena in X-ray Astronomy, Rome, p. 217
Swank J.H., Jahoda K., Zhang W., et al., 1995, The X-ray Timing Explorer: Progress and Science Prospects. In: Alpar M.A., Kiziloğlu Ü, van Paradijs J. (eds.) The Lives of the Neutron Stars, NATO ASI Ser. C 450, 523

Tanaka Y., Inoue H., Holt S.S., 1994, PASJ 46, L37

Trümper J., 1983, Adv. Space Res. 2, 241

Veigel W.M.J., Briggs E., Bates L., et al., 1971, X-ray Cross Section Compilation from $0.1 \mathrm{keV}$ to $1 \mathrm{MeV}$. Technical Report 2433F. Kaman Science Corporation, Colorado Springs, Co, U.S.A.

Weisskopf M.C., 1987, Ap. Lett. Comm. 26, 1

Wells A.A., Stewart G.C., Turner M.J.L., et al., 1992, SPIE 1574,205 